\title{
Conserved Tao Kinase Activity Regulates Dendritic Arborization, Cytoskeletal Dynamics, and Sensory Function in Drosophila
}

\author{
Chun Hu, ${ }^{1}$ Alexandros K. Kanellopoulos, ${ }^{2}$ Melanie Richter, ${ }^{3}$ Meike Petersen, ${ }^{1}$ Anja Konietzny, ${ }_{4}^{4}$ Federico M. Tenedini, ${ }^{1}$ \\ Nina Hoyer, ${ }^{1}$ Lin Cheng, ${ }^{1}$ Carole L.C. Poon, ${ }^{5}$ Kieran F. Harvey, ${ }^{5,6}$ Sabine Windhorst, ${ }^{7}$ Jay Z. Parrish, ${ }^{8}$ \\ @Marina Mikhaylova, ${ }^{4}$ Claudia Bagni, ${ }^{2,9}$ Froylan Calderon de Anda, ${ }^{3}$ and $\odot$ Peter Soba ${ }^{1}$ \\ ${ }^{1}$ Neuronal Patterning and Connectivity Laboratory, Center for Molecular Neurobiology (ZMNH), University Medical Center Hamburg-Eppendorf, 20251 \\ Hamburg, Germany, ${ }^{2}$ Department of Fundamental Neurosciences, University of Lausanne, 1005 Lausanne, Switzerland, ${ }^{3}$ Neuronal Development \\ Laboratory, Center for Molecular Neurobiology (ZMNH), University Medical Center Hamburg-Eppendorf, 20251 Hamburg, Germany, ${ }^{4}$ Neuronal Protein \\ Transport Laboratory, Center for Molecular Neurobiology (ZMNH), University Medical Center Hamburg-Eppendorf, 20251 Hamburg, Germany, ${ }^{5}$ Peter \\ MacCallum Cancer Centre, Melbourne, 3000 Victoria, Australia, ${ }^{6}$ Department of Anatomy and Developmental Biology, and Biomedicine Discovery \\ Institute, Monash University, Clayton, 3800 Victoria, Australia, ${ }^{7}$ Center for Experimental Medicine, University Medical Center Hamburg-Eppendorf, 20251 \\ Hamburg, Germany, ${ }^{8}$ Department of Biology, University of Washington, Seattle, 98195 Washington, and ${ }^{9}$ Department of Biomedicine and Prevention, \\ University of Rome Tor Vergata, 00133 Rome, Italy
}

Dendritic arborization is highly regulated and requires tight control of dendritic growth, branching, cytoskeletal dynamics, and ion channel expression to ensure proper function. Abnormal dendritic development can result in altered network connectivity, which has been linked to neurodevelopmental disorders, including autism spectrum disorders (ASDs). How neuronal growth control programs tune dendritic arborization to ensure function is still not fully understood. Using Drosophila dendritic arborization (da) neurons as a model, we identified the conserved Ste20-like kinase Tao as a negative regulator of dendritic arborization. We show that Tao kinase activity regulates cytoskeletal dynamics and sensory channel localization required for proper sensory function in both male and female flies. We further provide evidence for functional conservation of Tao kinase, showing that its ASD-linked human ortholog, Tao kinase 2 (Taok2), could replace Drosophila Tao and rescue dendritic branching, dynamic microtubule alterations, and behavioral defects. However, several ASD-linked Taok2 variants displayed impaired rescue activity, suggesting that Tao/Taok2 mutations can disrupt sensory neuron development and function. Consistently, we show that Tao kinase activity is required in developing and as well as adult stages for maintaining normal dendritic arborization and sensory function to regulate escape and social behavior. Our data suggest an important role for Tao kinase signaling in cytoskeletal organization to maintain proper dendritic arborization and sensory function, providing a strong link between developmental sensory aberrations and behavioral abnormalities relevant for Taok2-dependent ASDs.

Key words: autism spectrum disorders; cytoskeletal dynamics; dendritic arborization; sensory neuron; Tao kinase

Significance Statement

Autism spectrum disorders (ASDs) are linked to abnormal dendritic arbors. However, the mechanisms of how dendritic arbors develop to promote functional and proper behavior are unclear. We identified Drosophila Tao kinase, the ortholog of the ASD risk gene Taok2, as a regulator of dendritic arborization in sensory neurons. We show that Tao kinase regulates cytoskeletal dynamics, controls sensory ion channel localization, and is required to maintain somatosensory function in vivo. Interestingly, ASD-linked human Taok2 mutations rendered it nonfunctional, whereas its WT form could restore neuronal morphology and function in Drosophila lacking endogenous Tao. Our findings provide evidence for a conserved role of Tao kinase in dendritic development and function of sensory neurons, suggesting that aberrant sensory function might be a common feature of ASDs. 


\section{Introduction}

Developmental regulation of neuronal dendrite growth and branching, including the timing of growth events to maintain functionality of the nervous system, is essential for correct information processing of neuronal networks. Abnormal dendritic morphogenesis is thought to be a major contributing factor to the pathology of psychiatric and neurological disorders (Kulkarni and Firestein, 2012; Koleske, 2013; Chailangkarn et al., 2016). Accordingly, several genes linked to ASDs, including PTEN, MeCP2, and FMR1, have been described to affect dendritic arborization, resulting in either undergrown or overgrown dendritic fields (A. Lee et al., 2003; Kulkarni and Firestein, 2012; Huang et al., 2016). Moreover, recent evidence suggests that the disruption of ASD-linked genes, including MeCP2, CASPR2, GABRB3, and Shank3, can cause somatosensory neuron dysfunction, which contributes to behavioral defects, including anxiety-like behavior and nociceptive hyposensitivity or hypersensitivity (Han et al., 2016; Orefice et al., 2016, 2019; Dawes et al., 2018; Domínguez-Iturza and Bagni, 2019). Therefore, a better understanding of how sensory neuron development regulates and ensures function could provide direct insight into pathways involved in psychiatric and neurological conditions, such as ASDs.

Drosophila dendritic arborization (da) neurons in the PNS have served as an excellent model to investigate the mechanisms underlying dendritic development in vivo. da neurons extend sensory dendrites along the entire larval body wall and are classified according to their increasing dendritic complexity from Class I (C1da) to Class IV (C4da) (Grueber et al., 2002). C1da and C4da neurons exhibit very distinct dendritic growth properties during development: C1da neurons form most branches during late embryogenesis, which scale with animal size without considerable addition of new branches; C4da neurons, however, continue to elaborate higher-order branches throughout larval stages to maintain their space-filling receptive fields (Sugimura et al., 2003; Parrish et al., 2009; Hattori et al., 2013; Lin et al., 2015). Although several transcription factors and signaling molecules have been identified that are required for class-specific branching patterns (Grueber et al., 2003; Sugimura et al., 2004; JinushiNakao et al., 2007; Hattori et al., 2013; Ferreira et al., 2014; Yalgin et al., 2015), it has not been fully elucidated how dendritic arborization is achieved during development. Some of these cell

Received July 31, 2019; revised Jan. 13, 2020; accepted Jan. 16, 2020.

Author contributions: C.H. and P.S. designed research; C.H., A.K.K., F.M.T., N.H., and L.C. performed research; C.H., A.K.K., M.R., M.P., A.K., F.M.T., N.H., C.L.C.P., K.F.H., S.W., J.Z.P., M.M., C.B., and F.C.d.A. contributed unpublished reagents/analytic tools; C.H., A.K.K., F.M.T., L.C., and J.Z.P. analyzed data; C.H. wrote the first draft of the paper; C.H. and P.S. edited the paper; C.H. and P.S. wrote the paper.

A.K.K. and C.B. were supported by NCCR Synapsy 51NF40-158776 and SNSF 310030_182651/1. A.K.K. was supported by the Autism Speaks Meixner Postdoctoral Fellowship and Fondation Sophie Afenduli. A.K. and M.M. were supported by Deutsche Forschungsgemeinschaft Emmy-Noether Programm Grants MI 1923/1-1 and FOR2419 MI 1923/2-1. C.L.C.P. and K.F.H. were supported by National Health and Medical Research Council of Australia Project Grants APP1103604 and APP1142469. K.F.H. is a National Health and Medical Research Council Senior Research Fellow (APP1078220). J.Z.P. was supported by National Institutes of Health National Institute of Neurological Disorders and Sroke R01 NS076614. M.R. and F.C.d.A. were supported by Deutsche Forschungsgemeinschaft FOR 2419, CA1495/1-1, and CA 1495/4-1 and JPND (Bundesministerium für Bildung und Forschung, 01ED1806). F.C.d.A. and P.S. were supported by Landesforschungsförderung Hamburg LFF-FV27 and ERA-NET NEURON (Bundesministerium für Bildung und Forschung, 01EW1910). C.H. and P.S. were supported by Deutsche Forschungsgemeinschaft priority program SPP1926 (S01337/2-1). Stocks used in this study were obtained from the Bloomington Stock Center (National Institutes of Health P400D018537). We thank Buzz Baum for Tao plasmids; Ralf Pflanz for Tao antibody; Veit Riechmann for Tao ${ }^{\text {eta }}$ and UAS-Tao-HA flies; Zuoren Wang for ppk26 antibody; and Vittoria Mariano and Yuening Li for sharing preliminary data.

The authors declare no competing financial interests.

Correspondence should be addressed to Peter Soba at peter.soba@zmnh.uni-hamburg.de.

https://doi.org/10.1523/JNEUROSCI.1846-19.2020

Copyright $\odot 2020$ the authors type-specific programs have been shown to govern the expression of cytoskeletal regulators, which in turn shape the individual properties of actin and microtubule (MT) dynamics. Accordingly, cytoskeletal regulation is thought to be critically involved in dendrite growth and branching (Ori-McKenney et al., 2012; Stürner et al., 2019). To date, however, it is unclear how dendritic arborization and cytoskeletal dynamics are controlled during organismal growth to maintain sensory function.

Here we identified a conserved function for the Ste20-like kinase Tao in developmental growth control of dendritic arbors. We show that Tao is a negative regulator of dendrite arborization, MT dynamics, and F-actin localization. We further show that Tao activity affects mechanosensory channel localization and behavioral responses to noxious mechanical stimuli. The human Tao ortholog Taok2, which has recently been linked to ASDs (de Anda et al., 2012; Richter et al., 2019), can functionally rescue loss of Drosophila Tao phenotypes at the morphological, cytoskeletal, and behavioral level. We further show that several of its ASDlinked variants are functionally impaired, providing a link between dendrite growth, cytoskeletal regulation, and sensory function. Last, we show that Tao/Taok 2 are required in sensory neurons during development and in adult flies to maintain dendritic structure and somatosensory functions underlying escape and social behavior. Collectively, our study provides insight into the developmental regulation of dendrite growth by Tao kinase activity, linking cytoskeleton-driven growth and sensory function likely relevant for ASDs.

\section{Materials and Methods}

Fly stocks and transgenic constructs. All fly stocks were maintained at $25^{\circ} \mathrm{C}$ and $70 \%$ relative humidity on standard cornmeal/molasses food. All Drosophila stocks were obtained from Bloomington Drosophila Stock Center (Bloomington, IN), except if stated otherwise. The following alleles and transgenic lines were used: Tao ${ }^{\text {eta }}$,FRT19A (kindly provided by V. Riechmann) (Gomez et al., 2012), UAS-Tao-HA (kindly provided by V. Riechmann) (Gomez et al., 2012), Tao ${ }^{R N A i}$ (BDSC, stock \#35147), UAS-Tao-D168A (Sato et al., 2007), UAS-Tao-D423-900 (Poon et al., 2018), TrpA1 $1^{\text {MIO7645-GFSTF.O }}$ (BDSC, stock \#61795), Gal4 ${ }^{p p k}$, UASmCD4-tdTomato, UAS-mCD4-GFP, ppk-CD4-tdTomato (Han et al., 2011), Gal4 ${ }^{98 B}$ (Lin et al., 2015), UAS-EB1-GFP (Zheng et al., 2008), UAS-LifeAct-GFP (provided by J. Wildonger), Gal4 ${ }^{\text {nompC }}, 21-7-G a l 4$ (Cheng et al., 2010), and 5-40-Gal4 (Song et al., 2007). UAS-flag$h$ TaoK2 WT, A135P, A335V , and P1022* lines were generated by cloning the corresponding cDNAs with a C-terminal 3xflag-tag into the pUAST$A t t B$ vector. Position-specific transgenic lines were generated by embryo injection of attP16 (second chromosome) carrying flies (BestGene).

Mosaic Analysis with A Repressible Cell Marker (MARCM) analysis. For MARCM analyses of Tao mutants in da neurons, FRT19A (control) and Tao ${ }^{\text {eta }}, F R T 19 A$ virgins were crossed to TubP-Gal80,hs-flp,FRT ${ }^{19 A}$; Gal4 ${ }^{109(2) 80}, U A S-m C D 8-G F P$. Embryos were collected for $2 \mathrm{~h}$ at $25^{\circ} \mathrm{C}$ and allowed to develop for $3 \mathrm{~h}$ in yeast-plated agar plates, then heatshocked for $1 \mathrm{~h}$ at $37^{\circ} \mathrm{C}$. Heat-shocked embryos were kept at $25^{\circ} \mathrm{C}$, and da neuron MARCM clones were analyzed at third-instar larvae or specific time points. For dendritic tree quantification, only neurons located in segments A3-A7 were used.

Immunohistochemistry and antibodies. Larval filet preparation and staining were essentially performed as previously described (Soba et al., 2015). For endogenous Tao, phospho-Tao (pTao), human flag-Taok2, anti-GFP, and HRP immunostaining, larval filets were prepared in Ringer's buffer without calcium ( $130 \mathrm{~mm} \mathrm{NaCl}, 5 \mathrm{~mm} \mathrm{KCl,} 2 \mathrm{~mm} \mathrm{MgCl}$, 36 mm sucrose, 5 mm HEPES, pH 7.3). After 20 min fixation in 4\% formaldehyde/PBS, the samples were thoroughly washed in $0.3 \%$ Triton X-100/ PBS for 3 times. The samples were incubated with methanol at $-20^{\circ} \mathrm{C}$ for $10 \mathrm{~min}$ and washed briefly with $0.3 \%$ Triton X-100 in PBS for 3 times to remove methanol for Tao and pTao immunostaining. Then samples were transferred into blocking buffer containing $0.3 \%$ Triton X-100 in 
PBS with $10 \%$ normal goat serum for $1 \mathrm{~h}$ at room temperature. Anti-Tao (1:500) (Pflanz et al., 2015), anti-phospho-Tao (Ser181, 1:100, Santa Cruz Biotechnology, sc-135712, RRID:AB_2271461), anti-GFP (1:400, Invitrogen, A6455, RRID:AB_221570), and anti-flag (1:100, SigmaAldrich, F1804, RRID:AB_262044) antibodies were diluted and incubated in blocking buffer overnight at $4^{\circ} \mathrm{C}$. After washing, secondary DyLight or Alexa dye-conjugated donkey antibodies (1:400-1000) or Cy5-conjugated anti-HRP antibodies (1:500, Jackson ImmunoResearch Laboratories A, catalog \#123-095-021, RRID:AB_2314647) were incubated for $1 \mathrm{~h}$ at room temperature. After washing with $0.3 \%$ Triton X-100 in PBS three times for $5 \mathrm{~min}$, the samples were mounted in SlowFade Gold (Thermo Fisher Scientific) and prepared for imaging.

For anti-Futsch immunostaining, the procedures were the same as for Tao, except for the dissection buffer ( $120 \mathrm{~mm} \mathrm{NaCl}, 3 \mathrm{~mm} \mathrm{KCl}, 10 \mathrm{~mm}$ trehalose, $10 \mathrm{~mm}$ glucose, $10 \mathrm{~mm} \mathrm{NaHCO}$, $4 \mathrm{~mm} \mathrm{MgCl}_{2}, 10 \mathrm{~mm}$ sucrose, 10 mм HEPES, pH 7.25) during filet preparation. Anti-Futsch antibodies (22C10) were obtained from Developmental Studies Hybridoma Bank and used at 1:500.

For anti-ppk26 (gift from Zuoren Wang) immunostaining, the procedures and antibody were used as described previously (Guo et al., 2014). Secondary DyLight or Alexa-conjugated donkey antibodies were used at 1:400-1:1000 (Jackson ImmunoResearch Laboratories).

In vivo confocal microscopy. Drosophila da neurons were imaged by live confocal microscopy (Carl Zeiss, LSM700; or Olympus FV1000) of larvae mounted in a drop of $50 \%$ glycerol. Confocal stacks were taken to image C4da (ddaC) or C1da (ddaD) neuron dendrite fields either with a $20 \times /$ NA 0.8 air (full ddaC and ddaD fields) or $40 \times /$ NA 1.3 oil objectives (dorsal ddaC field). For the developmental imaging of the same $\mathrm{C} 1 \mathrm{da}$ or C4da neurons from 48 to $96 \mathrm{~h}$ after egg laying (AEL), each individual larva was imaged and put back on grape juice agar plates with fresh yeast paste and kept at $25^{\circ} \mathrm{C}$. The same neuron was imaged every $24 \mathrm{~h}$ during this time period, which allowed to trace and statistically analyze a specific neuron at all stages. The imaged larvae were allowed to develop to adulthood to ensure that handling and imaging did not interfere with normal development.

For adult C4da neuron imaging, mid-abdominal v'ada neurons from female flies (21-7-GAL4, UAS-CD4tdGFP;ppk-CD4-tdTomato) were imaged with a $20 \times /$ NA 0.8 air objective, which allowed visualization of the v'ada ventral dendritic field. For developmental analysis of UASTao ${ }^{\mathrm{RNAi}}$-induced changes in the dendritic field, 2-d-old adults were imaged. For adult-specific Tao manipulation, 21-7-GAL4, UASCD4tdGFP;ppk-CD4-tdTomato flies were crossed with a tubulin promoter-driven temperature-sensitive GAL80 (McGuire et al., 2003) with or without $U A S-T a o^{\mathrm{RNAi}}$. Animals were kept at $18^{\circ} \mathrm{C}$ (permissive temperature) and were shifted to $29^{\circ} \mathrm{C}$ (nonpermissive temperature) $2 \mathrm{~d}$ after eclosion to induce $T a o^{\mathrm{RNAi}}$ expression. Animals were imaged $5 \mathrm{~d}$ later on day 7 after eclosion.

Analysis of dendrite length, crossing, field coverage, and complexity. Dendrites of da neurons were traced with the Imaris Filament Tracer module (BitPlane) using confocal stacks. The parameters of dendritic length and number of terminals were automatically calculated by the software. Data for Sholl and reverse Strahler order analysis were extracted from the original data using Imaris. For consistency, larval da neurons from dorsal segments A4-A6 were imaged and statistically analyzed. For adult C4da neuron analysis, the ventral dendritic field of v'ada neurons from segments A4-A6 was analyzed and quantified as performed for larval da neurons.

Live imaging of EB1 dynamics. All imaging of da neurons was performed on intact larvae as previously described (Stewart et al., 2012) with modifications. For main branches, neurons were imaged using a $40 \times$ oil/NA 1.3 objective on a Carl Zeiss confocal microscope (LSM700, $512 \times 512$ pixel resolution in free-run mode). To avoid fluorescence bleaching and damaging of neurons, the pinhole size was increased, and laser power was minimized to capture most dendrites within a single optical section. To minimize variability that is usually present when analyzing EB1 dynamics (Stone et al., 2012; Nguyen et al., 2014; Yalgin et al., 2015; Sears and Broihier, 2016; Tao et al., 2016), we imaged all genotypes (2-5 genotypes) for each independent experiment in parallel (within $6-8 \mathrm{~h}$ ) using the same parameters to generate consistent data. In exper- iments with manipulation of Tao activity, EB1 dynamics in main branches were imaged in C4da ( $1 \mathrm{~s} /$ frame) and C1da $(0.8 \mathrm{~s} /$ frame $)$ neurons for $3 \mathrm{~min}$. For terminal branches in $\mathrm{C} 4 \mathrm{da}$ neurons, to improve the resolution and quality of the images, a $40 \times / \mathrm{NA} 1.3$ oil objective (with zoom set to $1 \times$ ) was used. Images were acquired with maximum scan speed for a total 120 frames (1.56 s/frame) in free-run mode (187 s/experiment). Terminal branches, which showed readily identifiable tips, were used for analysis. Movies were analyzed using ImageJ (National Institutes of Health). EB1 comets were detected by the Image Kymograph plugin, and an EB1-labeled comet was counted only if it was detectable and tracked in consecutive frames for $>5 \mathrm{~s}$. The number of EB1 comets was quantified and data presented as EB1 number per $100 \mu \mathrm{m}$ per $1 \mathrm{~min}$. The speed of EB1 was measured using Particle Tracker (Fiji, ImageJ, National Institutes of Health, http://cmci.embl.de/documents/ 121005advancedimg).

Analysis of LifeAct expression levels and distribution. The dorsal field of C4da ddaC neurons expressing LifeAct-GFP with $p p k$-Gal4 $>C D 4$ tdTomato was imaged with confocal microscopy at the third instar larval stage (96-108 h AEL). Acquired stacks were then processed with Fiji/ Image (National Institutes of Health) to normalize the LifeAct-GFP signal to tdTomato expression using a previously described procedure (Kardash et al., 2011; Soba et al., 2015). Briefly, maximum projections of 2-color stacks were used, and alignment of the tdTomato and LifeActGFP channels using the TurboReg plugin (ImageJ) was performed. The LifeAct-GFP signal was thresholded automatically (ImageJ) and normalized to tdTomato levels using the RatioPlus plugin (ImageJ). For LifeActGFP quantification along the dendritic arbors, normalized intensity values were obtained by ROI tracing of the branches using the PlotProfile function (ImageJ, $n=10$ per genotype).

Quantitative immunohistochemistry of ppk26, TrpA1, and Futsch. To compare protein expression levels in C4da neurons, larvae of different genotypes in the same experimental groups were processed simultaneously. Larval filets of $w^{1118}$ or animals with C4da neuron-specific expression ( $p$ pk-Gal4>CD4-tdGFP) of Tao ${ }^{\mathrm{RNAi}}$ and Tao ${ }^{\mathrm{FL}}$ were prepared and immunostained as described above. The same settings for image acquisition were applied to all experimental groups, and signal saturation was minimized. Futsch and ppk26 signal intensity in C4da neuron dendrites was quantified as described above for the analysis of LifeAct-GFP (Soba et al., 2015).

A line carrying a GFP exon trap in the endogenous TrpAl genomic locus (TrpA1 $1^{\text {MIO7645-GFSTF.O }}$ ) was used to visualize the resulting $\mathrm{N}$-terminally GFP-tagged TrpA1, which should reflect expression and localization of endogenous TrpA1. The effect of Tao overexpression or RNAi on TrpA1 MI07645-GFSTF. ${ }^{\text {M }}$ levels was examined by immunohistochemistry using anti-GFP and anti-HRP antibodies. The same settings for image acquisition were applied to all experimental groups, and signal saturation was minimized. The specific GFP signal intensity was quantified, and anti-HRP signal was used as a reference.

Behavioral assays. Mechanonociceptive assays were performed as previously described (Hu et al., 2017; Hoyer et al., 2018). Briefly, a $45 \mathrm{mN}$ mechanical force was given to the dorsal side (abdominal segment A3A5) of freely moving third instar larvae ( $96 \pm 3 \mathrm{~h} \mathrm{AEL}$ ) twice within $2 \mathrm{~s}$. Only a complete $360^{\circ}$ rolling response was counted as nociceptive rolling, and responses to the second stimulus are shown. Experiments were performed in a blinded and randomized fashion with $>65$ larvae per genotype.

For negative geotaxis (elicited escape response), adult male flies (maximum 7-d-old, females for Tao ${ }^{\text {eta/+ }}$ experiments) were collected and placed in individual $14 \mathrm{ml}$ polystyrene tubes as described previously (Kosmidis et al., 2011). Observation of negative geotaxis was performed in a dark room using infrared light. The polystyrene tubes were vortexed and tapped on a surface to knock down the fly to the bottom part of the tube. The time for each fly to climb a certain distance was measured twice and the average calculated. The time of at least 16 flies per genotype was measured, and the results were analyzed parametrically for statistical significance using the GraphPad Prism 5 software.

For social interaction, 4- to 7-d-old male flies (females for Tao ${ }^{\text {eta/+ }}$ experiments) were separated in tubes of 8 each, $24 \mathrm{~h}$ before the assay. On the day of the assay, flies were placed without anesthesia in empty tubes 
without food for $1 \mathrm{~h} 30 \mathrm{~min}$. After the starvation, flies were exposed to a food droplet for 2 min to acclimate to this disturbance. The flies were then observed for $2 \mathrm{~min}$, and the number of social interactions (including lunges, aggression and courtship) between them was counted.

For adult-specific perturbation of Tao, GAL4 $^{\text {elav }}$ was combined with tubulin promoter-driven temperature-sensitive GAL80 (McGuire et al., 2003). For GAL4 ${ }^{\text {elav }} ;$ tub$G A L 80^{t s}$ experiments, all crosses were cultured at $18^{\circ} \mathrm{C}$, and expression was induced specifically in adult flies by shifting the temperature to $29^{\circ} \mathrm{C} 2 \mathrm{~d}$ after eclosion.

Experimental design and statistical analysis. For all experiments, controls and genetically matched experimental genotypes were processed in parallel. The experimental design ensured that all experimental groups were used in a balanced way throughout the entire experiment. Specific genders were used for adult behavior (males only, except for Tao ${ }^{\text {eta/+ }}$ females for heterozygous Tao mutant analyses) and adult sensory neuron morphology (females), mixed genders were randomly used for all larval experiments. No statistical methods were used to predetermine sample sizes, but sample sizes were chosen to be similar to those reported in previous publications (Zheng et al., 2008; Dankert et al., 2009; Stewart et al., 2012; Hattori et al., 2013; Ferreira et al., 2014; Mauthner et al., 2014; Lin et al., 2015; Sears and Broihier, 2016). Neuron or animal numbers are indicated in the figure legends. Origin Pro (Origin Labs) or Prism 8 (GraphPad Software) was used for statistical analysis. The data were presented as individual data points with mean (long line) and \pm SE (short lines). A two-tailed unpaired Student's $t$ test was used for comparing two groups. One-way-ANOVA with Bonferroni post hoc test was used for comparing three or more groups, and Welch's test was used if the data are unequal variances. Two-way ANOVA with Tukey post hoc was used for comparing three or more groups in Sholl analysis. Mann-Whitney test or Student's $t$ test (for two groups) and Kruskal-Wallis test with Dunn's post hoc test for multiple comparisons were used for adult and larval mechanonociceptive behavioral analysis. $p<0.05$ was considered statistically significant.

\section{Results}

Tao is a negative regulator of dendritic arborization

To identify signals controlling dendritic arborization during larval development, we screened a collection of cytoskeletal regulators by using RNA interference (RNAi) and putative mutant alleles covering 53 genes for effects on dendritic development. We examined the dendritic morphology of $\mathrm{C} 4$ da neurons at the midthird instar larval stage (ddaC, $\sim 96 \mathrm{~h} \mathrm{AEL}$ ) and found that 7/53 genes (Ank2, dlc90F, hiw, klp10A, par-1, patronin, pdk1) caused dendritic growth reduction. Only one gene (Tao kinase) resulted in dendritic overbranching and overgrowth (Fig. 1-1, available at https://doi.org/10.1523/JNEUROSCI.1846-19.2020.f1-1). Drosophila Tao is highly conserved and belongs to the Ste20-like kinase family with three vertebrate orthologs (Dan et al., 2001). To confirm our screening results, we analyzed a Tao-null mutant unpaired Student's $t$ test).

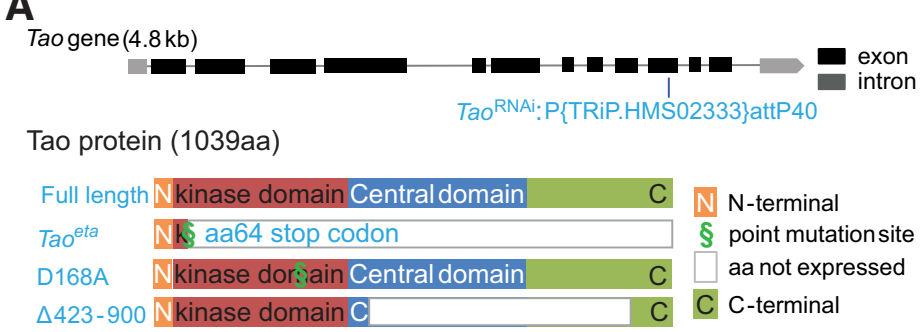

C4da

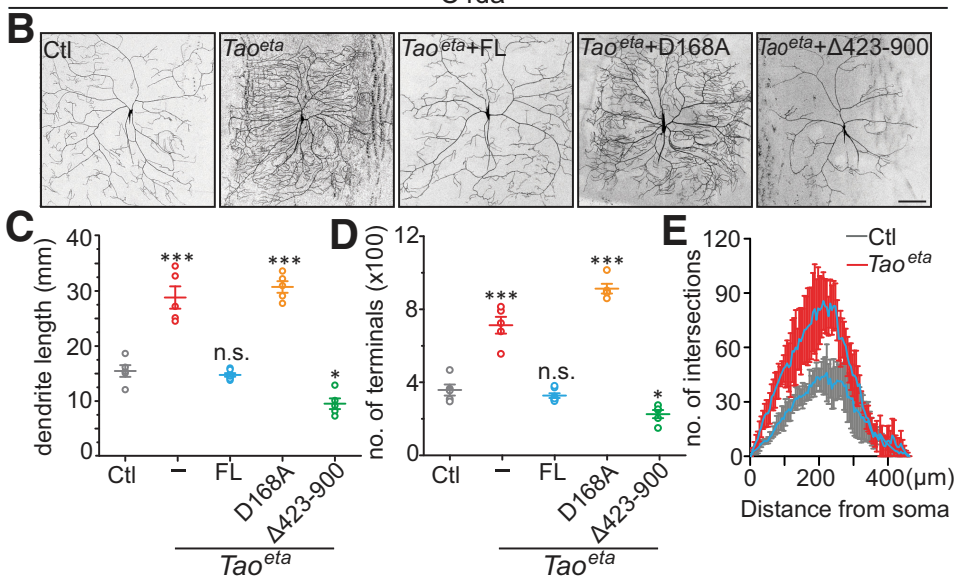

C1da

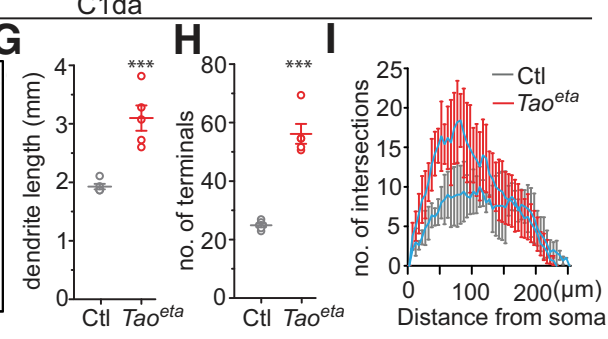

Figure 1. Tao is a negative regulator of dendritic arborization in da sensory neurons. $\boldsymbol{A}$, Schematic diagram of the Tao exonintron structure, protein domain organization, Tao mutant alleles, and transgenic constructs (N/C: N/C terminus, point mutations, deletion gaps, and domain distribution; full-length: WT Tao; Tao eta: Tao-null mutant; D168A: kinase-inactive form; $\Delta 423-900$ : ive form). B, MARCM analysis of dendritic phenotypes of Tao-null mutant (4da neurons without and with overexpression of different transgenic Tao constructs as indicated. Scale bar, $100 \mu \mathrm{m}$. $\boldsymbol{C}-\boldsymbol{E}$, Analysis of total dendrite length (C), number of terminals (D), and dendritic complexity by Sholl analysis (mean \pm SD) $(\boldsymbol{E})$ of $T a 0^{\text {eta }}$ mutant C4da neurons $(n=5, n=5, n=7$, control (Ctl) and Tao eta mutant C1da neurons. $\mathbf{G}-\boldsymbol{I}$, Analysis of total dendrite length $(\boldsymbol{G})$, number of terminals $(\boldsymbol{H})$, and dendritic complexity by Sholl analysis (I) of Tao ${ }^{\text {eta }}$ mutant C1da neurons ( $n=5 /$ genotype). ${ }^{* * *} p<0.001$ compared with Ctl (two-tailed

allele (Tao ${ }^{\text {eta }}$ ) (Gomez et al., 2012) using MARCM (T. Lee and Luo, 2001) to assess its cell-autonomous function in dendrite development (Fig. 1A). Tao mutant C4da neurons showed dramatic dendritic overgrowth; in the absence of Tao function, C4da neurons exhibited a nearly twofold increase in overall dendritic length, terminal numbers, and complexity compared with controls (Fig. 1B-E; one-way ANOVA, dendrite length: $F_{(4,22)}=$ $66.158, p=6.08 \times 10^{-12}$; Bonferroni post hoc test, $p=4.904 \times$ $10^{-7}, 1,4.889 \times 10^{-8}, 0.016$, dendrite terminals: $F_{(4,22)}=$ $105.349, p=5.140 \times 10^{-14}$; Bonferroni post hoc test, $p=1.512 \times$ $\left.10^{-7}, 1,3.604 \times 10^{-11}, 0.039\right)$. This overgrowth was not accompanied by an expansion in dendrite territory. Instead, dendrite arbors occupied comparable receptive field areas with increased complexity, but without obvious dendritic self-avoidance or tiling defects. The Tao mutant dendritic overbranching phenotype could be completely rescued by expressing full-length Tao $\left(\mathrm{Tao}^{\text {eta }+} \mathrm{Tao}^{\mathrm{FL}}\right)$ but not a kinase-inactive Tao transgene $\left(\mathrm{Tao}^{\text {eta }+}\right.$ 

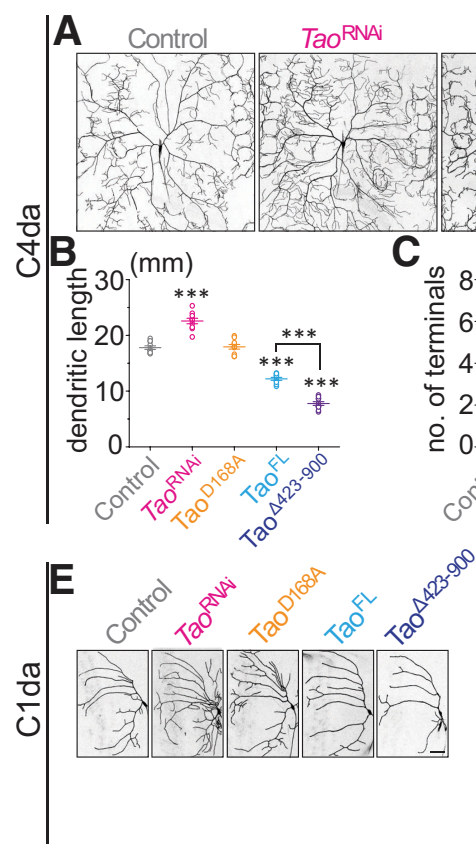

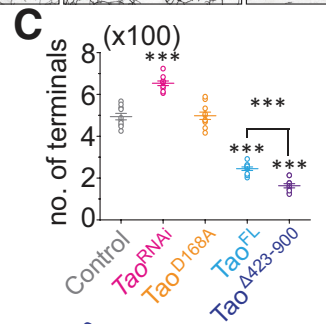

$\mathbf{F}_{E^{(m) * *}}(\mathrm{~mm})^{* *}$

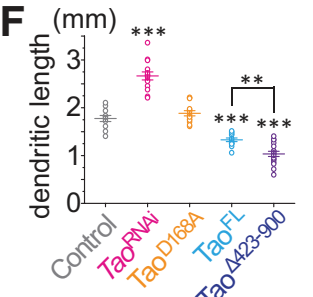

人o
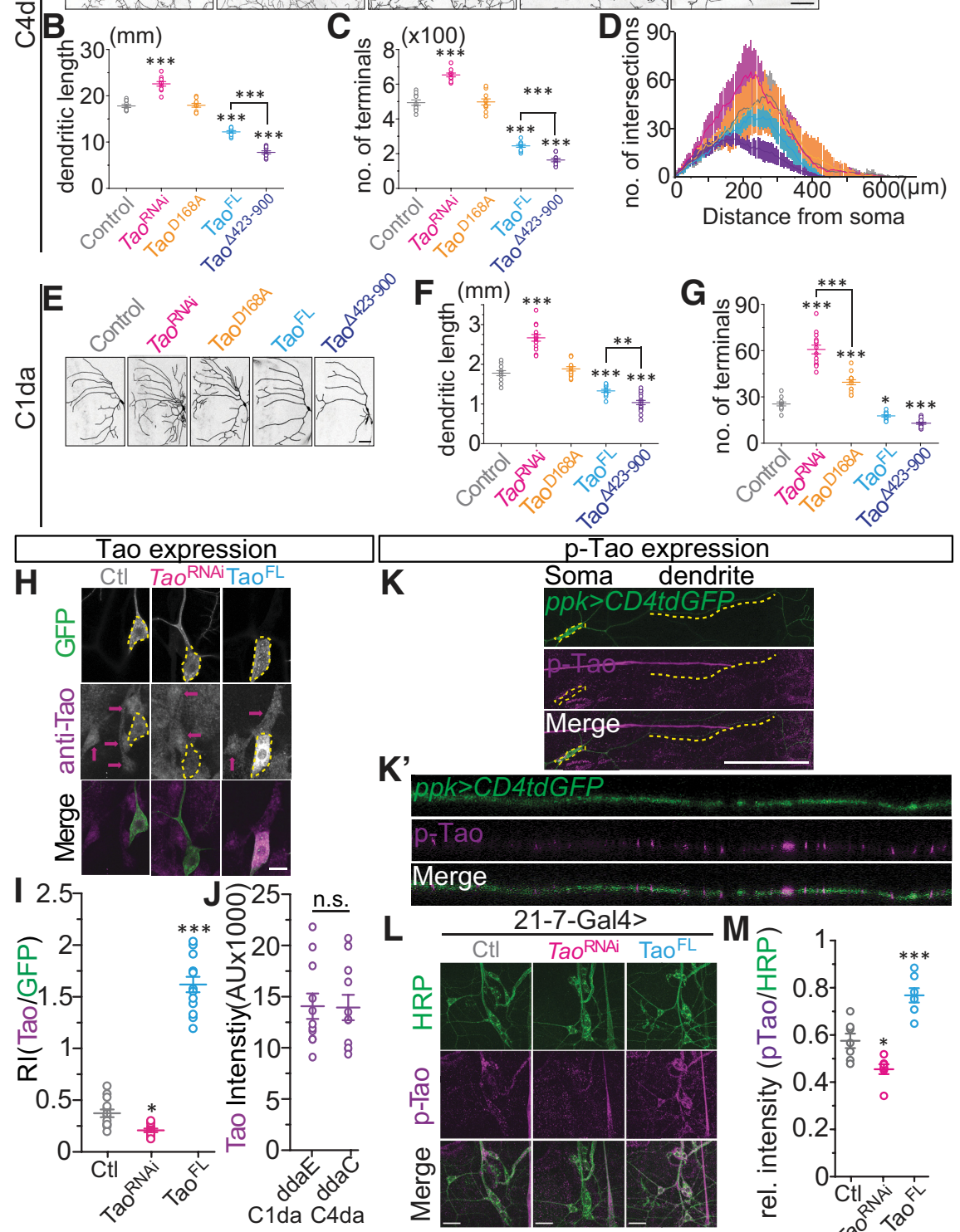

$\mathbf{K}^{\prime}$
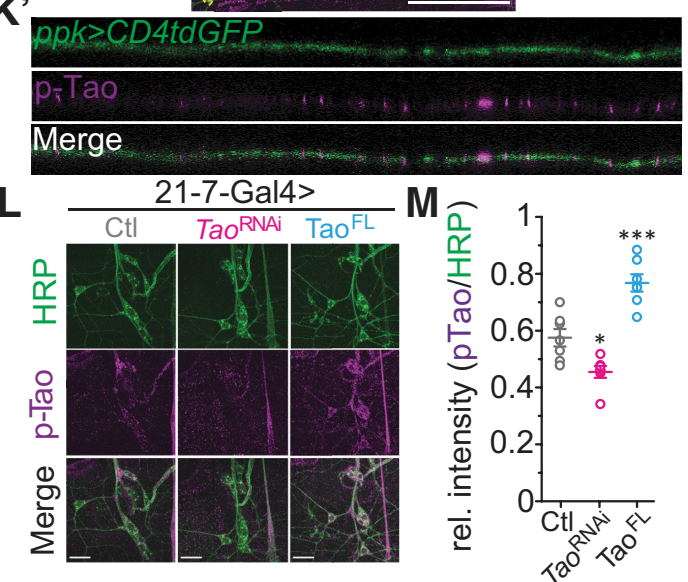

Figure 2. Tao controls dendritic arborization of da neurons in a kinase activity-dependent manner and is endogenously expressed in da sensory neurons. $\boldsymbol{A}$, Representative images of dendritic phenotypes after manipulation of Tao activity in (4da neurons (ppk-Gal4, UAS-CD4-tdGFP). Scale bar, $100 \mu \mathrm{m}$. $\boldsymbol{B}-\boldsymbol{D}$, Quantitative analysis of dendritic length ( $\boldsymbol{B}$ ), terminal numbers $(\boldsymbol{C})$, and dendrite complexity by Sholl analysis $(\boldsymbol{D})$ of the indicated genotypes ( $n=10$ /genotype). ${ }^{* *} p<0.01,{ }^{* * *} p<0.001$ compared with Ctl (one-way ANOVA with Bonferroni post hoc test). E-G, Analogous analysis of C1da neuron morphology after Tao kinase perturbation (98B-Gal4, UAS-CD4-tdGFP) with quantification of dendritic length $(\boldsymbol{F})$ and terminal numbers $(\boldsymbol{G})(n=11$, $n=14, n=14, n=13, n=17) .{ }^{* *} p<0.01,{ }^{* * *} p<0.001$ compared with (tl (one-way ANOVA with Bonferroni post hoc test). $\boldsymbol{H}$, (4da neurons ( $\mathrm{ddaC}$ ) in third instar larvae visualized by Gal4 ${ }^{\text {ppk }}$-driven mCD4-tdGFP and anti-Tao immunostaining under control, $T a 0^{R N A i}$, or Tao overexpression $\left(T a 0^{F L}\right)$ conditions. Purple arrows indicate somata. Yellow dotted lines indicate C4da soma. Scale bar, $10 \mu \mathrm{m}$. I, Relative fluorescence intensity of anti-Tao signals normalized to GFP ( $n=13$ neurons from $>5$ animals). ${ }^{* *} p<0.01,{ }^{* * *} p<0.001$ compared with Ctl (one-way ANOVA with Bonferroni post hoc test). J, Mean intensity of anti-Tao signal in C4da ( $\mathrm{ddaC}$ ) and (1da (ddaE) neurons ( $n=11$ for each genotype from at least 6 larvae). $\boldsymbol{K}$, Endogenous anti-p-Tao immunostaining in third instar larval filets with (4da neurons labeled by CD4-tdGFP expression. Yellow dotted lines indicate (4da neuron soma and dendrites. $\boldsymbol{K}^{\prime}$, (4da neuron dendrite portion resliced in Z direction (as shown in $\boldsymbol{D}$ ) and displaying discrete dendritically localized p-Tao puncta. Scale bar, $50 \mu \mathrm{m}$. $L$, Manipulation of Tao activity levels by Tao ${ }^{\mathrm{RNAi}}$ or Tao overexpression in da neurons using 21-7-Gal4 and anti-p-Tao immunostaining. da neurons were visualized by anti-HRP. Tao ${ }^{\text {RNAi }}$ reduces p-Tao levels, whereas Tao overexpression increases p-Tao levels in da neurons. Scale bar, $20 \mu \mathrm{m}$. $\boldsymbol{M}$, Quantitative analysis of p-Tao levels in da neuron somata normalized to HRP signals ( $n=7 /$ genotype). ${ }^{*} p<0.05,{ }^{* *} p<0.001$ compared with Ctl (one-way ANOVA with Bonferroni post hoc test).
$\mathrm{TaO}^{\mathrm{D} 168}$ ) specifically in C4da neurons (Fig. $1 A-D)$. Conversely, expression of a hyperactive form of Tao $\left(\right.$ Tao $\left.^{\text {eta }}+\mathrm{TaO}^{\Delta 423-900}\right)$ significantly reduced dendritic branching. Finally, we found that Tao mutation similarly affected other sensory neurons, including C1da (Fig. $1 F-I$; unpaired $t$ test, Fig. $1 G$ : $t_{(8)}=-5.296, p=7.323 \times 10^{-4}$; Fig. $1 H: t_{(8)}=-8.988, p=1.871 \times 10^{-5}$ ) and C3da neurons (C3da dendritic length: Ctl: $4.11 \pm 0.46$, Tao $^{\text {eta }}: 6.40 \pm$ 0.37. unpaired $t$ test, $t_{(4)}=-3.886, p=$ 0.018. $n=3$ /genotype), by increasing branching and growth of the entire dendritic arbor.

To further address the importance of Tao kinase activity in dendrite development, we knocked down (Tao ${ }^{\mathrm{RNAi}}$; Fig. $1 A$ ) or overexpressed Tao with altered kinase activity selectively in C4da or C1da neurons. In C4da neurons, knockdown of Tao (ppk-Gal4) resembled the Tao mutant phenotype displaying exuberant dendritic branching, albeit to a lower extent (Fig. 2A-D; one-way ANOVA: Fig. 2B: $F_{(4,45)}=236.801, p=0$, Bonferroni post hoc test, vs Ctl: $1.064 \times 10^{-10}, 1,7.597 \times$ $10^{-13}, 3.985 \times 10^{-22}$; FL vs $\Delta 423-900$ : $p=9.687 \times 10^{-10}$; Fig. $2 C: F_{(4,45)}=$ 241.907, $p=0$, Bonferroni post hoc, vs Ctl: $3.212 \times 10^{-10}, 1,1.528 \times 10^{-16}, 3.039 \times$ $10^{-21}$; FL vs $\Delta 423-900: p=5.345 \times$ $\left.10^{-4}\right)$. While overexpression of kinasedead Tao (Tao ${ }^{\mathrm{D} 168 \mathrm{~A}}$ ) had no obvious effect on C4da dendrite morphogenesis, full-length $\left(\mathrm{Tao}^{\mathrm{FL}}\right.$ ) or hyperactive Tao (Tao ${ }^{\Delta 423-900}$ ) expression resulted in significantly decreased dendritic branching, with the latter displaying more dramatically reduced dendritic branches (Fig. 2A$D)$. Comparable dendrite phenotypes were detected upon Tao manipulation in C1da neurons (98B-Gal4), except that expression of kinase-dead Tao showed a partial loss-of-function effect, suggesting it might act in a dominantnegative fashion (Fig. 2E-G; one-way ANOVA, Fig. $2 F: F_{(4,64)}=111.485, p=$ 0 , Bonferroni post hoc test, vs Ctl: $2.695 \times 10^{-13}, 1,1.011 \times 10^{-4}, 5.463 \times$ $10^{-11}$; FL vs $\Delta 423-900: p=0.008$; Fig. $2 G: F_{(4,64)}=149.019, p=0$, Bonferroni post hoc test, vs Ctl: $4.246 \times 10^{-21}$, $1.970 \times 10^{-6}, 0.023,1.986 \times 10^{-5}$; Tao $^{\mathrm{RNAi}}$ vs D168A: $p=1.196 \times 10^{-12}$, FL vs $\Delta 423-900: p=0.532$ ).

Using immunohistochemistry, we confirmed that Tao is expressed in all da neurons and that RNAi-mediated knockdown or Tao overexpression indeed decreased or increased its levels, respectively (Fig. 2H-J; Fig. 2I: one-way ANOVA, $F_{(2,36)}=242.642, p=0$, Bonferroni post 


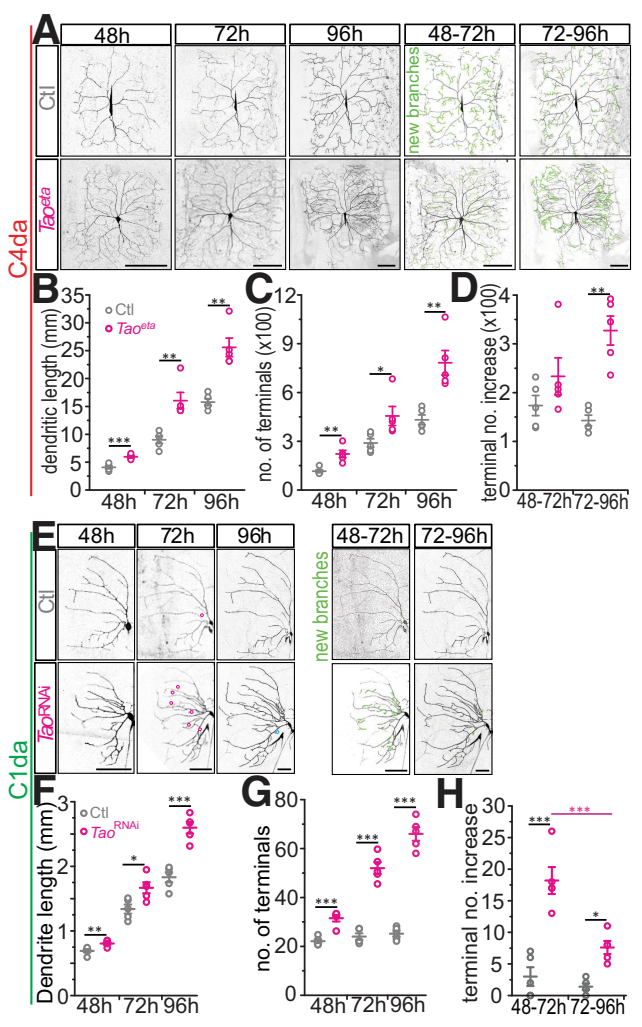

Figure 3. Tao developmentally limits dendritic arborization in da sensory neurons. A-D, Developmental analysis of ( 4 da neurons dendritic morphogenesis with MARCM. $\boldsymbol{A}$, Representative images showing dendritic development of the same Control (CtI) and $T a 0^{\text {eta }}$ mutant $\mathrm{C} 4 \mathrm{da}$ neuron from 48 to $96 \mathrm{~h} \mathrm{AEL}$. Green represents the new branches generated during different time windows. Scale bar, $100 \mu \mathrm{m} . \boldsymbol{B}, \boldsymbol{C}$, Quantitative comparison of dendritic length $(\boldsymbol{B})$ and terminal numbers $(\boldsymbol{C})$ of $\mathrm{Ctl}$ and $\mathrm{TaO}^{\text {eta }}$ (4da neurons from 48 to $96 \mathrm{~h}$ AEL. The Tao ${ }^{\text {eta }}$ phenotype was already visible at $48 \mathrm{~h} \mathrm{AEL}$ ( $n=5 /$ genotype). ${ }^{*} p<0.05,{ }^{* *} p<0.01,{ }^{* * *} p<0.001$ compared with Ctl (two-tailed unpaired Student's $t$ test). $D$, The number of newly added terminals during the different time points are shown ( $n=5$ /genotype). ${ }^{* *} p<0.01$ (two-tailed unpaired Student's $t$ test). $\boldsymbol{E}-\boldsymbol{H}$, Developmental analysis of (1da neuron dendritic morphogenesis using $T a 0^{R N A i}$. $\boldsymbol{E}$, Representative images showing dendritic development of the same Control and $T a 0^{R N A i}$ C1da neurons from 48 to $96 \mathrm{~h} \mathrm{AEL}$. Red and blue circles represent new branches generated from $48-72 \mathrm{~h}$ and $72-96 \mathrm{~h} \mathrm{AEL}$, respectively. Green represents the new branches generated during different time windows. Scale bar, $50 \mu \mathrm{m}$. $\boldsymbol{F}, \mathbf{G}$, Quantitative comparison of dendritic length $(\boldsymbol{F})$ and terminal numbers $(\boldsymbol{G})$ of control and $T a 0^{R N A i}(1 \mathrm{da}$ neurons from 48 to $96 \mathrm{~h} \mathrm{AEL}$ ( $n=5 /$ genotype). ${ }^{*} p<0.05,{ }^{* *} p<0.01,{ }^{* * *} p<0.001$ compared with Ctl (twotailed unpaired Student's $t$ test). $\boldsymbol{H}$, The number of newly added terminals during the different time points are shown ( $n=5 /$ genotype). ${ }^{*} p<0.05,{ }^{* * *} p<0.001$ (one-way ANOVA with Bonferroni post hoc test).

hoc test, $p=0.024,2.033 \times 10^{-19}$; Fig. $2 \mathrm{~J}$ : unpaired $t$ test, $t_{(20)}=$ $0.0753, p=0.941)$. Moreover, using a phospho-specific antibody against the active form of Tao (p-Tao), we could detect p-Tao puncta throughout the $\mathrm{C} 4 \mathrm{da}$ dendritic arbor (Fig. $2 \mathrm{~K}$ ). As with total Tao, p-Tao levels were also reduced or elevated upon Tao ${ }^{\mathrm{RNAi}}$ or Tao overexpression, respectively (Fig. $2 \mathrm{~L}, \mathrm{M}$; one-way ANOVA, $F_{(2,18)}=32.522, p=1.056 \times 10^{-6}$, Bonferroni post hoc test, $\left.p=0.020,3.283 \times 10^{-4}\right)$. This suggests that the active form of Tao can be efficiently upregulated or downregulated and that Tao kinase activity is necessary and sufficient to limit dendritic branching and growth of sensory neurons.

Tao activity controls dendritic arborization and growth dynamics

We next asked at which time point Tao functions to limit dendritic arborization. To this end, we imaged the same da neuron throughout development from 48 to $96 \mathrm{~h} \mathrm{AEL}$. Tao ${ }^{\eta}$ mutant
C4da neurons already displayed exuberant arborization at $48 \mathrm{~h}$ AEL (Fig. $3 A-C$; unpaired $t$ test, Fig. $3 B: 48$ h: $t_{(8)}=-5.762, p=$ $4.234 \times 10^{-4} ; 72 \mathrm{~h}: t_{(8)}=-4,355, p=0.002 ; 96 \mathrm{~h}: t_{(8)}=-5.508$, $p=5.680 \times 10^{-4}$; Fig. $3 C: 48$ h: $t_{(8)}=-4.381, p=0.002 ; 72 \mathrm{~h}:$ $\left.t_{(8)}=-2.599, p=0.032 ; 96 \mathrm{~h}: t_{(8)}=-4.321, p=5.680 \times 10^{-4}\right)$, and a progressive increase of terminal numbers from 72 to $96 \mathrm{~h}$ AEL compared with control (Fig. 3D; one-way ANOVA, $F_{(3,16)}=$ 9.213, $p=8.945 \times 10^{-4}$, Bonferroni post hoc, $\left.{ }^{* *} p=0.001\right)$. Next, we investigated $\mathrm{C} 1 \mathrm{da}$ neurons, which grow during larval development by extending existing branches rather than adding new branches, allowing us to detect aberrantly generated dendrites at late time points (Sugimura et al., 2003; Yalgin et al., 2015). Using C1da neuron-specific knockdown of Tao $\left(\mathrm{Tao}^{R N A i}\right)$, we observed continuous branch addition throughout all larval stages (Fig. $3 E-H$; unpaired $t$ test: Fig. $3 F$ : 48 h: $t_{(8)}=-3.399, p=9.37 \times$ $10^{-3}$; $72 \mathrm{~h}: t_{(8)}=-2.981, p=0.0176$; $96 \mathrm{~h}: t_{(8)}=-6.507, p=$ $1.868 \times 10^{-4}$; Fig. $3 G: 48$ h: $t_{(8)}=-6.462, p=1.958 \times 10^{-4}$; $72 \mathrm{~h}: t_{(8)}=-10.146, p=7.617 \times 10^{-6} ; 96 \mathrm{~h}: t_{(8)}=-11.333, p=$ $3.310 \times 10^{-6}$; one-way ANOVA, Fig. $3 H: F_{(3,16)}=28.445, p=$ $1.196 \times 10^{-6}$, Bonferroni post hoc test, $48-72 \mathrm{~h}: p=6.744 \times$ $10^{-6}, 72-96 \mathrm{~h}: p=0.0423$, Tao $^{\mathrm{RNAi}} 48-72 \mathrm{~h}$ vs $72-96 \mathrm{~h}: 4.482 \times$ $\left.10^{-4}\right)$. These results show that, after loss of Tao, even C1da neurons regain the ability to generate new branches during larval stages. Collectively, these results provide evidence that Tao kinase function is limiting dendritic arborization throughout larval development.

To understand how Tao affects dendritic branch dynamics, we performed short-term time-lapse imaging in third instar larvae. First, we imaged the same C4da neuron at two time points ( 0 and $30 \mathrm{~min}$ ) to quantify dendritic growth changes within this timeframe. In control larvae, $\sim 35 \%$ of dendrite terminals were extending, 30\% were retracting, and 35\% were stable (Fig. $4 A, B$ ). Tao overexpression ( $\mathrm{Tao}^{\mathrm{FL}}$ ) resulted in very similar percentages of dendrite extension and retraction compared with control. However, $\mathrm{Tao}^{\mathrm{RNAi}}$ in C4da neurons resulted in an increased percentage $(50 \%)$ of growing terminals at the expense of retracting (20\%) and stable (30\%) branches (Fig. 4A, B; one-way ANOVA, $F_{(2,9)}=10.581, p=4.33 \times 10^{-3}$, Bonferroni post hoc test, ${ }^{* *} p=$ $\left.9.17 \times 10^{-3}\right)$. This resulted in a twofold increase in dendritic terminal growth/retraction ratios for Tao ${ }^{\mathrm{RNAi}}$ compared with $\mathrm{Tao}^{\mathrm{FL}}$ or control C4da neurons (Fig. $4 C$; one-way ANOVA, $F_{(2,9)}$ $=15.353, p=1.26 \times 10^{-3}$, Bonferroni post hoc, $\left.{ }^{* *} p=0.0023\right)$. These results suggest that loss of Tao-induced overgrowth is in part due to a selective increase in terminal branch stabilization and growth.

To get a more precise insight into dendrite turnover, which might explain the overall growth defect upon Tao overexpression, we performed a detailed analysis by imaging C4da neurons every $5 \mathrm{~min}$ for $30 \mathrm{~min}$. We quantified dendrite extension and retraction speed and found that both $T a o^{\mathrm{RNAi}}$ and, in particular $\mathrm{Tao}^{\mathrm{FL}}$, significantly decreased dendrite dynamics (Fig. 4D-F; one-way ANOVA, Tao ${ }^{\mathrm{RNAi}}$ extension: $F_{(2,543)}=18.342, p=$ $1.958 \times 10^{-8}$, Bonferroni post hoc test, $p=0.005$; retraction: $F_{(2,608)}=23.278, p=1.814 \times 10^{-10}$, Bonferroni post hoc test, $p=$ $5.219 \times 10^{-4}$, aao $^{\mathrm{FL}}$ extension: $p=1.33 \times 10^{-8}$; retraction: $p=$ $\left.7.408 \times 10^{-11}\right)$. This suggests that increased Tao activity induced by overexpression deregulates branch growth by slowing down dynamics rather than altering branch growth/retraction rates. Together, these results suggest that Tao kinase regulates dendrite development by controlling dynamic dendrite growth and retraction through regulation of branch stability and dynamics. 


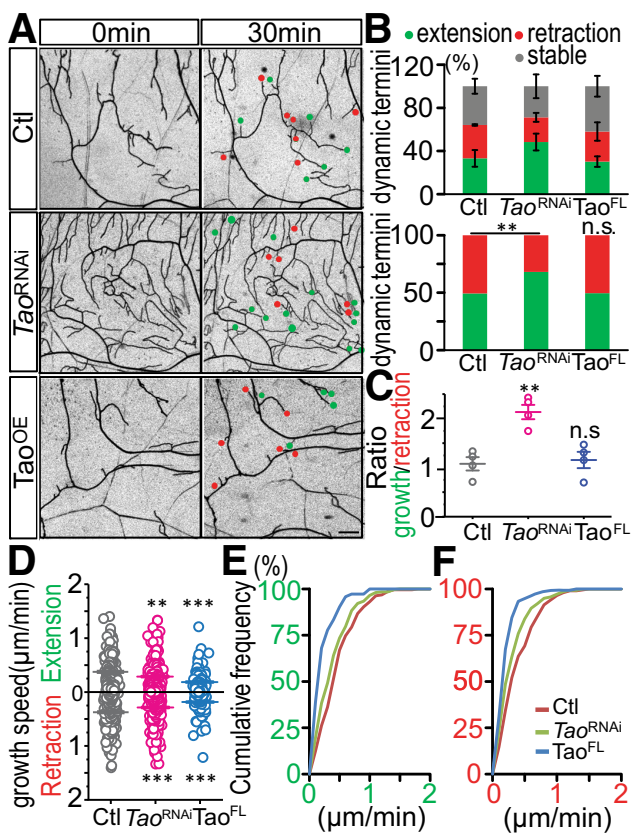

Figure 4. Tao affects dendritic terminal dynamics. $A$, Dendritic terminal dynamics of C4da $\mathrm{ddaC}$ neurons in third instar ( $\sim 96 \mathrm{~h} \mathrm{AEL}$ ) larvae were time-lapse imaged every 5 min over a 30 min interval. Representative images show dendritic terminal dynamics at 0 and 30 min in $\mathrm{Ctl}$, $\mathrm{Tao}^{\mathrm{RNAi}}$, and Tao ${ }^{\mathrm{FL}}$ (4da neurons. B, Quantification of terminal branch extension (green), retraction (red), and stable terminals (gray). C, Quantification of ratios between extension and retraction. $\boldsymbol{D}$, Quantification of terminal branch extension (positive value) and retraction (negative value) speed revealed by time-lapse confocal images over $5 \mathrm{~min}$ intervals. Growth speed is reduced for $T a 0^{\mathrm{RNAi}}$ and even more for $\mathrm{TaO}^{\mathrm{FL}}$ expression. $\boldsymbol{E}, \boldsymbol{F}$, Cumulative frequency of growth speed for extension $(\boldsymbol{E})$ and retraction $(\boldsymbol{F})(n=80, n=61, n=60$ terminals from 4 neurons/genotype). ${ }^{* *} p<0.01,{ }^{* * *} p<0.005$ compared with $\mathrm{Ctl}$ (Mann-Whitney $U$ test). Scale bar, $20 \mu \mathrm{m}$.

\section{Tao kinase activity specifically controls the number of dynamic MTs}

To characterize the cellular mechanism of these growth defects, we investigated effects of Tao kinase on the neuronal cytoskeleton. Tao has been implicated in the regulation of MT dynamics in vitro, which are a critical driving force for dendritic branching and growth (Timm et al., 2003; Johne et al., 2008; Liu et al., 2010; King and Heberlein, 2011). We thus tested whether downregulation or upregulation of Tao activity affected dendritic MT dynamics in $\mathrm{C} 1 \mathrm{da}$ and $\mathrm{C} 4 \mathrm{da}$ neurons. Individual growing MTs were tracked by time-lapse imaging using the plus-end binding protein EB1 tagged with GFP (EB1-GFP) (Satoh et al., 2008; Zheng et al., 2008; Stewart et al., 2012). UAS-EB1-GFP was expressed using $\mathrm{Gal4}^{p \text { pk }}$ (C4da) or Gal4 ${ }^{\text {nompC }}$ (C1da), and we determined the number, directionality/polarity, and speed of dendritic EB1 comets using kymographs in both main and terminal branches (Fig. $5 A, B)$.

To analyze C4da neuron dendritic MT dynamics in detail, we investigated both proximal $(0-160 \mu \mathrm{m}$ from soma) and distal (160-320 $\mu \mathrm{m}$ from soma) dendrites separately (Fig. $5 A$ ). We found a higher abundance of EB1 comets in proximal than in distal dendrites, but we did not observe significant differences in polarity and speed at $96 \mathrm{~h}$ AEL. Strikingly, Tao knockdown $\left(\mathrm{Tao}^{\mathrm{RNAi}}\right)$ increased the number of dynamic MTs, while overexpression of either full-length $\left(\mathrm{TaO}^{\mathrm{FL}}\right.$ ) or hyperactive Tao $\left(\mathrm{TaO}^{\Delta 423-900}\right.$ ) significantly decreased the number of dynamic MTs in the proximal region and overall in C4da neuron dendrites (Fig. $5 B, C$; one-way ANOVA, proximal: $F_{(4,45)}=12.43, p=$ $6.846 \times 10^{-7}$, Welch's test, $p=0.0004, p=0.0925, p=0.131$, $p=0.0039 ;$ distal: $F_{(4,45)}=6.471, p=3.346 \times 10^{-4}$, Welch's test, $p=0.0188, p=0.0615, p=0.3401, p=0.3295$; overall: $F_{(4,45)}=$ 14. 657, $p=9.695 \times 10^{-8}$, Welch's test, $p=0.0004, p=0.0949$, $p=0.0084, p=0.0028)$. Loss of Tao did not affect overall MT polarity or speed in any of the analyzed genotypes (Fig. $5 D$, E; Fig. $5 D$ : one-way ANOVA, MT polarity: $F_{(4,45)}=0.467, p=0.759$; Fig. 5E: MT speed: Ctl: $1.412 \pm 0.019$, Tao $^{\mathrm{RNAi}}: 1.250 \pm 0.017$, D168A: $1.337 \pm 0.014$, FL: $1.244 \pm 0.040, \Delta 423-900: 1.165 \pm$ $0.021, \mu \mathrm{m} / 10 \mathrm{~s})$. We also examined the effect of Tao on MT dynamics in C4da neuron terminal branches, which harbor a higher proportion of anterograde MTs that have been linked to dendrite extension (Ori-McKenney et al., 2012; Yalgin et al., 2015). Tao ${ }^{\mathrm{RNAi}}$ in C4da neurons increased the percentage and abundance of dendrite terminals containing EB1 comets compared with control (Fig. $5 F, G$; unpaired $t$ test, Fig. $5 F: t_{(34)}=$ $-2.515, p=0.017$, Fig. $\left.5 G: t_{(473)}=-2.432, p=0.015\right)$. However, the overall number, polarity, and speed of dynamic MTs in each individual terminal were not affected (Fig. $5 H-J$; unpaired $t$ test, Fig. $5 H: t_{(214)}=-0.618, p=0.537$, Fig. $5 I: t_{(34)}=-0.653, p=$ 0.518, Fig. 5J: $\left.t_{(416)}=0.291, p=0.771\right)$.

We further investigated Tao-dependent MT regulation in C1da neurons at $72 \mathrm{~h}$ AEL, allowing us to visualize dynamic MTs in the entire dendritic tree. Consistent with our findings in C4da neurons, C1da neurons displayed significantly elevated numbers

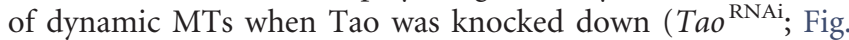
$5 K, L$; unpaired $t$ test, Fig. $5 L$ : neuron: $t_{(28)}=-3.041, p=0.0051$, $\left.100 \mu \mathrm{m}: t_{(28)}=-4.456, p=1.225 \times 10^{-4}\right)$. Most dynamic MTs originated from different sites in control and Tao ${ }^{\text {RNAi }} \mathrm{Clda}$ neurons, with the latter displaying a strong increase in additional putative nucleation sites, possibly due to an increase in local nucleators, MT catastrophe rescue, and/or increased severing of MTs (Fig. $5 \mathrm{~K}, M$, magenta and yellow dots; unpaired $t$ test, $\left.t_{(28)}=-4.326, p=1.741 \times 10^{-4}\right)$. As for C4da neurons, the polarity and speed of dynamic MTs were unaltered in Tao RNAi C1da neurons (Fig. $5 \mathrm{~N}, \mathrm{O}$; Fig. $5 \mathrm{~N}$ : unpaired $t$ test, $t_{(28)}=$ $-0.904, p=0.374$, Fig. 5O: Ctl: $1.457 \pm 0.045$, Tao ${ }^{\mathrm{RNAi}}$ : $1.610 \pm 0.041, \mu \mathrm{m} / 10 \mathrm{~s})$

To address whether the observed increase in dynamics MTs might also alter MT stability, we analyzed the abundance of the Map1b homolog futsch, which binds to MTs and is a marker of stabilized MTs required for dendrite growth (Hummel et al., 2000; Stewart et al., 2012). Tao knockdown or overexpression in C4da neurons affected Futsch levels by increasing or decreasing its abundance in dendrites, respectively (Fig. 6; one-way ANOVA, $F_{(2,26)}=19.949, p=5.615 \times 10^{-6}$, Bonferroni post hoc test, $p=0.011, p=0.019)$. This suggests that overall MT stability is inversely correlated with Tao activity.

Collectively, these data show that Tao regulates the number, but not the polarity or speed, of dynamic MTs in a kinase activitydependent manner. Consistent with our analysis of dendrite dynamics, the increase in dynamic MT numbers and Futsch/Map1b abundance upon loss of Tao suggest that they contribute to stability and growth of dendrites.

\section{Tao activity alters dendritic F-actin abundance and localization}

F-actin is intimately linked to MTs dynamics as well as dendrite arborization (Konietzny et al., 2017; Zhao et al., 2017; Stürner et al., 2019). Since Tao has also been implicated in the regulation of F-actin in cultured cells (Johne et al., 2008), we investigated the effect of altered Tao activity on F-actin localization. F-actin in C4da neurons was directly visualized in vivo by expressing Lifeact-GFP as in previous reports (Ferreira et al., 2014; Soba et 
al., 2015; Ori-McKenney et al., 2016). Normalized Lifeact-GFP signal along major C4da neuron dendrites was fairly evenly distributed, while terminal branches typically featured hotspots of increased F-actin levels in C4da neurons. Tao ${ }^{\text {RNAi }}$ expression in C4da neurons significantly increased relative $\mathrm{F}$-actin levels, and F-actin was strongly enriched in main and terminal branches without altering the overall distribution ratio (Fig. $7 A-D$; oneway ANOVA, Fig. $7 B$ : $F_{(4,36)}=8.517, p=$ $6.07 \times 10^{-5}$; Bonferroni post hoc test: $p=1.772 \times 10^{-5}, p=0.159, p=0.071$, $p=0.933$; Fig. $7 C: F_{(4,36)}=9.331, p=$ $2.79 \times 10^{-5}$; Bonferroni post hoc test: $p=$ 0.018, $p=0.828, p=1.011 \times 10^{-5}$, $p=0.082$; Fig. $7 D: F_{(4,36)}=9.412, p=$ $2.589 \times 10^{-5}$; Bonferroni post hoc test: $p=1, p=1, p=6.108 \times 10^{-4}, p=$ $0.009)$. In contrast, overexpression of active $\left(\mathrm{Tao}^{\mathrm{FL}}\right.$ or $\mathrm{Tao}^{\Delta 423-900}$ ), but not kinase-impaired Tao in C4da neurons resulted in altered distribution of F-actin with a relative increase in dendrite terminals. Overall, these data suggest that Tao activity affects F-actin levels and distribution in dendrites, which likely contributes to the observed dendrite arborization effects.

\section{Tao function alters sensory channel localization}

Sensory function relies on dendrite morphogenesis and cytoskeletal integrity to ensure proper sensory channel transport and localization. As Tao function affects dendrite arborization as well as cytoskeletal dynamics, we wondered whether this might affect functional expression and/or localization of sensory channels in this system. Several mechanosensory channels in C4da neuron dendrites are involved in the detection of mechanonociceptive stimuli, including $\operatorname{TrpA} 1, \operatorname{ppk} 1 / \mathrm{ppk} 26$, and Piezo (Zhong et al., 2010; Kim et al., 2012; Gorczyca et al., 2014; Guo et al., 2014).

We first analyzed surface expression and distribution of endogenous ppk26 using a specific antibody (Guo et al., 2014). Using nonpermeabilizing conditions, ppk26 was only localized to dendrites but not to the soma or axon in agreement with previous reports (Gorczyca et al., 2014; Guo et al., 2014). Interestingly, ppk26 channels were unevenly distributed on dendrites compared with the continuous distribution of anti-HRP signal labeling all neuronal membranes (Fig. 8A). Overall, similar distribution patterns were also detected in C4da neurons expressing Tao ${ }^{\mathrm{RNAi}}$ or $\mathrm{TaO}^{\mathrm{FL}}$. We then quantified ppk26 intensity distribution by normalizing and thresholding it to the anti-HRP signal (for details, see Materials and Methods). The resulting ppk26 coverage index (dendritic length labeled with ppk26 divided by corresponding total dendritic length) served as a measure of ppk26 surface ex(unpaired Student's $t$ test).

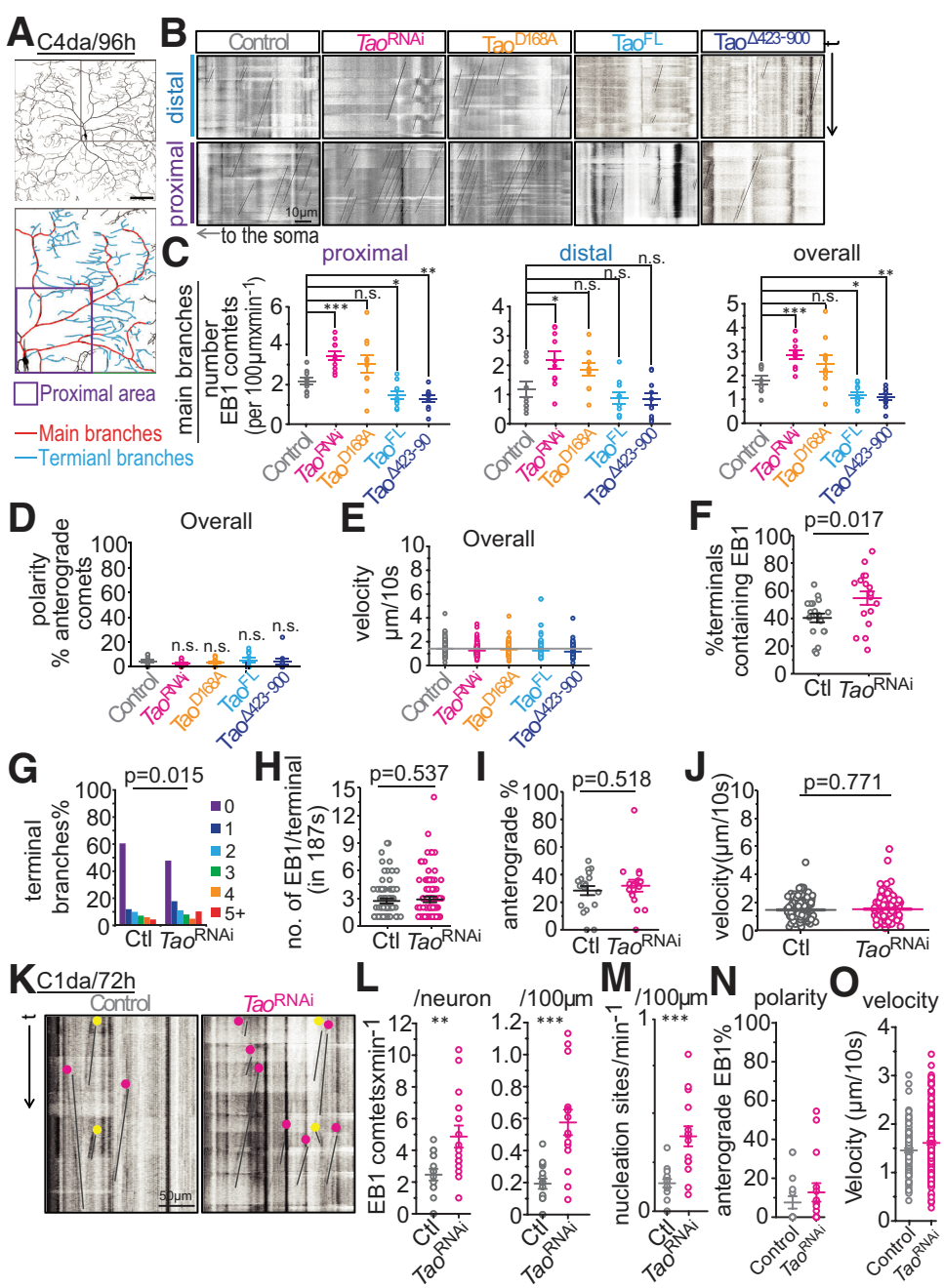

Figure 5. Tao kinase activity regulates dendritic MT dynamics. $A-J$, MT dynamics in C4da neurons. $A$, Schematic image showing a C 4 da neuron at $96 \mathrm{~h}$ AEL. The main branches (red) and terminal dendrites (blue) are labeled. The proximal dendrites were defined as $0-160 \mu \mathrm{m}$ (purple box), distal dendrites as $160-320 \mu \mathrm{m}$ from the soma. $\boldsymbol{B}$, Representative kymographs showing EB1-GFP comets in control or after manipulation of Tao kinase activity by specific overexpression of indicated Tao transgenes in (4da neurons ( $p p k$-Gal4). Scale bar, $10 \mu \mathrm{m}$. $\boldsymbol{C}-\boldsymbol{E}$, Quantitative data showing the number $(\boldsymbol{C})$, polarity $(\boldsymbol{D})$, and velocity $(\boldsymbol{E})$ of EB1-GFP comets in proximal or distal dendrites and overall comparison ( $n=10$ /genotype). ${ }^{*} p<0.05,{ }^{* *} p<0.01,{ }^{* * *} p<0.001$ compared with $\mathrm{Ctl}$ (one-way ANOVA with Welch test). $\boldsymbol{F}$-J, For analysis of MT dynamics in terminal branches, only dendrites showing no additional higher order branches were defined as terminal dendrites. The percentage of terminals containing EB1 $(\boldsymbol{F})$, the distribution of the number of comets observed per branch $(\boldsymbol{G})$, the number of EB1 in each terminal branch $(\boldsymbol{H})$, polarity of MTs in each terminal $(\boldsymbol{I})$, and velocity of dynamic MTs $(\boldsymbol{J})$ are shown. Exact $p$ values are indicated (Student's $\boldsymbol{t}$ test). $\boldsymbol{K}-\boldsymbol{O}$, MT dynamics ual comets originating from a single nucleation site. Scale bar, $50 \mu \mathrm{m}$. $\mathbf{L}-\mathbf{0}$, Quantitative data showing the EB1 comet numbers $(\boldsymbol{L})$, number of nucleation sites $(\boldsymbol{M})$, polarity $(\boldsymbol{N})$, and velocity $(\boldsymbol{O})\left(n \geq 15 /\right.$ genotype). ${ }^{* *} p<0.01,{ }^{* * *} p<0.001$

pression and coverage (Fig. $8 B$ ). In main branches (primary and secondary dendrites), a small but significant decrease in the ppk26 coverage index was observed in $\mathrm{Tao}^{\mathrm{FL}}$ but not $\mathrm{Tao}^{\mathrm{RNAi}}$ expressing C4da neurons compared with control (Fig. $8 C$; oneway ANOVA, $F_{(2,178)}=8.589, p=2.749 \times 10^{-4}$; Bonferroni post hoc test: $\left.p=0.679,2.229 \times 10^{-4}\right)$. Both Tao ${ }^{\mathrm{FL}}$ and Tao ${ }^{\mathrm{RNAi}}$ expression increased the relative intensity of ppk26 on main branches (Fig. $8 D$; one-way ANOVA, $F_{(2,178)}=10.565, p=$ $4.628 \times 10^{-5}$; Bonferroni post hoc test: $p=0.0013,1.064 \times$ $\left.10^{-4}\right)$. In terminals, however, only knockdown of Tao resulted in a significant decrease in ppk26 coverage index as well as intensity, suggesting that Tao ${ }^{\mathrm{RNAi}}$ disrupts and impedes ppk26 distribution 


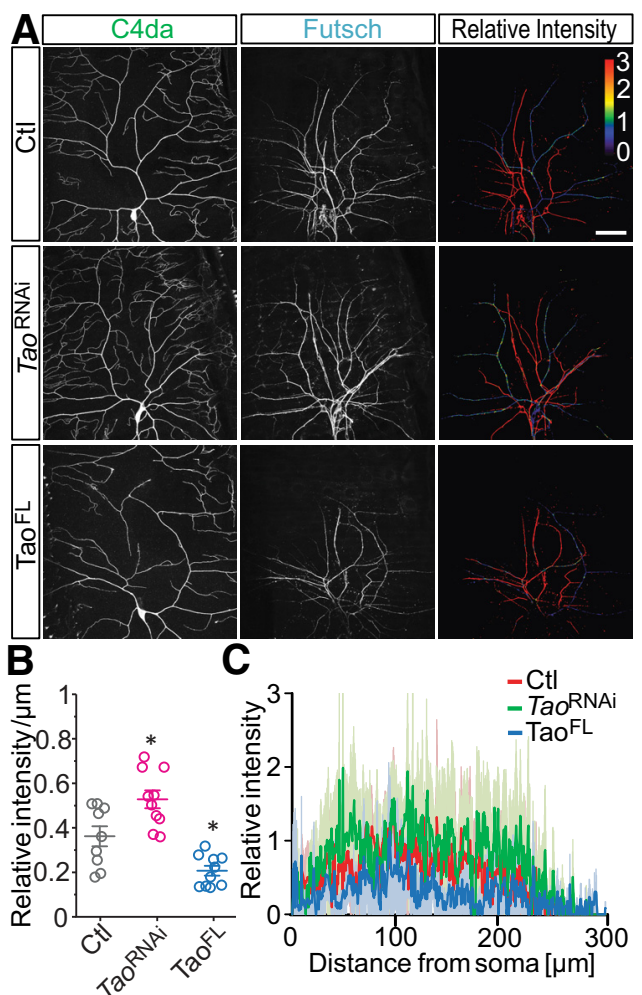

Figure 6. Tao regulates Futsch/Map1b levels and distribution in C4da neurons. A, Comparison of Futsch-positive MTs in WT (Ctl), Tao ${ }^{\text {RNAi }}$-and Tao ${ }^{\mathrm{FL}}$-expressing C4da da neurons. Endogenous Futsch (anti-Futsch (22c10): 1:100) is detected in all da neurons. The relative intensity of Futsch normalized to CD4::tdGFP expressed only in C4da neurons is shown. The high-intensity (red) dendrites are from non-C4da neurons. Scale bar, $50 \mu \mathrm{m}$. B, Quantification of relative Futsch intensities $(n=9, n=10, n=10) .{ }^{*} p<0.05$ (one-way ANOVA with Bonferroni correction). C, Normalized Futsch signal along the longest primary dendrites was plotted against increasing soma distance showing the different intensity profiles of $\mathrm{Ctl}, \mathrm{TaO}^{\mathrm{RNAi}}$, and $\mathrm{Ta}^{\mathrm{FL}} \mathrm{C}$ (dda neurons. Solid lines indicate mean levels. Shaded area represents SD.

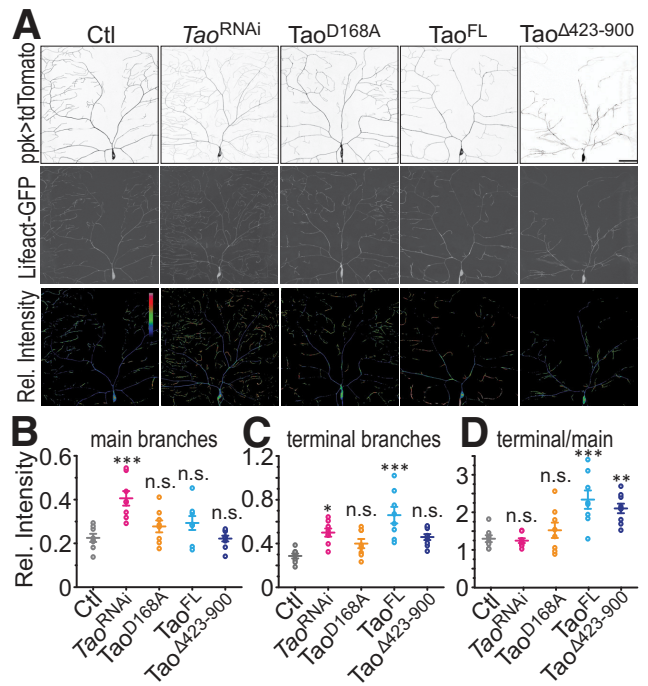

Figure 7. Tao affects F-actin levels and distribution in C4da neurons. F-actin was visualized by Lifeact-GFP in C4da neurons labeled by CD4::tdTomato. $\boldsymbol{A}$, Representative images of C4da neurons expressing Lifeact-GFP and relative intensity normalized to mCD4::tdTomato. $\boldsymbol{B}-\boldsymbol{D}$, Quantification of relative Lifeact-GFP intensity in $(\boldsymbol{B})$ main branches, $(\boldsymbol{C})$ terminals, and $(\boldsymbol{D})$ terminal to main branch ratios. Scale bar, $50 \mu \mathrm{m} . n \geq 8 .{ }^{*} p<0.05,{ }^{* *} p<0.01,{ }^{* * *} p<0.001$ compared with Ctl (one-way ANOVA with Bonferroni correction). in terminal branches (Fig. $8 E-G$; one-way ANOVA, Fig. $8 E$ : $F_{(2,355)}=30.209, p=7.66 \times 10^{-13}$; Bonferroni post hoc test: $p=$ $1.096 \times 10^{-11}$; Fig. $8 G: F_{(2,355)}=12.935, p=3.78 \times 10^{-6}$; Bonferroni post hoc test: $p=0.004,0.124)$.

We then used an allele labeling endogenous TrpA1 with GFP to assess whether it is also affected by Tao perturbation. Due to the low levels, we could only visualize somatic TrpA1 in C4da neurons (Fig. $8 H$ ). However, we observed a strong decrease in TrpA1-GFP immunoreactivity upon $\mathrm{Tao}^{\mathrm{FL}}$ or Tao ${ }^{\mathrm{RNAi}}$ expression, suggesting that Tao activity has a profound effect on TrpA1 abundance (Fig. 8 H,I; one-way ANOVA, $F_{(2,33)}=13.579$, $p=$ $4.978 \times 10^{-5}$; Bonferroni post hoc test: $p=8.726 \times 10^{-4}$, $8.243 \times 10^{-5}$ ). Together, these results suggest that Tao activity regulates the localization and abundance of mechanosensory channels in C4da neurons.

\section{Human TaoK2, but not ASD-linked, variants rescue loss of} Tao induced anatomical, cytoskeletal, and behavioral defects We next assessed whether the observed changes in structure, cytoskeletal dynamics, and mechanosensory channel localization result in functional defects. Moreover, we wanted to address whether Drosophila Tao and its closest mammalian homolog of Drosophila Tao, Tao Kinase 2 (TaoK2), are functionally conserved. TaoK2 has been previously implicated in basal dendrite formation of pyramidal neurons (de Anda et al., 2012). In addition, human TaoK2 is located on chromosome 16p11.2, a major susceptibility locus for ASDs (Weiss et al., 2008) and schizophrenia (McCarthy et al., 2009). A recent study further established $\mathrm{hTaoK} 2$ as an ASDs risk gene, showing that a patient-derived de novo mutation (A135P) impairs TaoK2 kinase activity and function in vivo (Richter et al., 2019). Additional alleles were reported in this study, including A335V (not affecting TaoK2 activity) and P1022*, with the latter resulting in a premature stop codon and altered TaoK2 localization and activity. We thus assayed the ability of TaoK2 and its ASD-linked variants to rescue loss of Tao function phenotypes in C4da neurons.

Morphologically, $\mathrm{Tao}^{\mathrm{RNAi}}$-induced dendritic overbranching of C4da neurons could be fully rescued by hTaoK2 ${ }^{\mathrm{WT}}$ expression (Fig. $9 A-C$; one-way ANOVA, Fig. $9 B: F_{(4,45)}=24.105, p=$ $1.057 \times 10^{-10}$; Bonferroni post hoc test: vs Ctl: $p=4.370 \times 10^{-8}$, $1,6.288 \times 10^{-7}, 1$; vs $\mathrm{Tao}^{\mathrm{RNAi}}: 3.283 \times 10^{-7}, 1,2.337 \times 10^{-6}$; WT vs A135P: $4.743 \times 10^{-6}$; WT vs A335V: 1 ; A135P vs A335V: $3.318 \times 10^{-5}$; Fig. 9 C: $F_{(4,45)}=13.715, p=2.172 \times 10^{-7}$; Bonferroni post hoc test: vs Ctl: $p=1.298 \times 10^{-4}, 1,1.135 \times 10^{-4}, 1$; vs Tao ${ }^{\mathrm{RNAi}}$ : $0.002,1,5.445 \times 10^{-5}$; WT vs A135P: 0.002 ; WT vs A335V: 1 ; A135P vs A335V: $4.753 \times 10^{-5}$ ). In contrast, misexpression of the kinase-impaired ASD-linked TaoK2 variant (hTaoK2 ${ }^{\mathrm{Al} 35 \mathrm{P}}$ ) failed to rescue the dendritic phenotype, despite identical expression levels. While the A335V variant showed full rescue activity, hTaoK2 ${ }^{\mathrm{P} 1022 *}$ overexpression could not fully restore normal arborization $\left(\mathrm{P}_{1022^{*}}\right.$ rescue dendritic length: $\mathrm{Ctl}$ : $17.45 \pm 2.52$, P1022* OE: $16.44 \pm 6.24$, Tao $^{\mathrm{RNAi}}: 22.98 \pm 4.85$, $\mathrm{Tao}^{\mathrm{RNAi}}+\mathrm{P} 1022^{*}$ OE: $20.70 \pm 5.56, \mathrm{~mm}$; one-way ANOVA, $F_{(3,36)}=36.26, p=5.64 \times 10^{-11}$; Bonferroni post hoc: vs Ctt: $0.964,1.629 \times 10^{-8}, 2.921 \times 10^{-4} ; \mathrm{Tao}^{\mathrm{RNAi}}$ vs Tao ${ }^{\mathrm{RNAi}}+\mathrm{P} 1022^{*}$ OE: 0.016; number of terminals: Ctl: $364 \pm 10, \mathrm{P}_{1022^{\star}} \mathrm{OE}: 403 \pm$ 17, $\mathrm{Tao}^{\mathrm{RNAi}}: 505 \pm 16, \mathrm{Tao}^{\mathrm{RNAi}}+\mathrm{P} 1022^{*} \mathrm{OE}: 510 \pm 20$; one-way ANOVA, $F_{(3,36)}=20.15, p=7.769 \times 10^{-8}$; Bonferroni post hoc: vs Ctl: $0.557,2.869 \times 10^{-6}, 1.553 \times 10^{-6} ; \mathrm{Tao}^{\mathrm{RNAi}}$ vs $\mathrm{Tao}^{\mathrm{RNAi}}+\mathrm{P} 1022^{*} \mathrm{OE}: 1, n=10$ /genotype).

We then assayed whether hTaoK2 could also restore MT dynamics. Consistent with the dendritic phenotypes, hTaoK2 ${ }^{\mathrm{WT}}$ and hTaoK2 ${ }^{\mathrm{A} 335 \mathrm{~V}}$, but not hTaoK2 ${ }^{\mathrm{Al} 35 \mathrm{P}}$, overexpression could 
restore the number of dynamic MTs to control levels (Fig. 9D; one-way ANOVA, Fig. 9B: $F_{(4,55)}=6.461, p=2.458 \times 10^{-4}$; Bonferroni post hoc test: vs Ctl: $p=0.006$, 1, 0.032, 1; vs Tao ${ }^{\mathrm{RNAi}}$ : $\left.0.041,1,0.003\right)$. These results imply that hTaoK2 and Drosophila Tao have conserved kinase activity-dependent functions in cytoskeletal regulation and dendrite development.

We then asked whether the observed changes in arborization, cytoskeleton, and mechanosensory channel localization induced by the loss of Tao alter the function of C4da neurons and whether hTaoK2 could ameliorate behavioral defects as well. C4da neurons are primary nociceptors responding to harsh mechanical touch, which results in a stereotyped rolling escape response (Tracey et al., 2003; Zhong et al., 2010; Hu et al., 2017). We performed mechanonociception assays using a calibrated von Frey filament (45 $\mathrm{mN}$ ) and assayed third instar larvae (96 h AEL) expressing Tao ${ }^{\mathrm{RNAi}}$ specifically in C4da neurons. Compared with controls, Tao knockdown in C4da neurons resulted in a strong impairment of nociceptive rolling responses (Fig. 9E, $E^{\prime}$; KruskalWallis test, $\mathrm{H}=37.67, p<0.0001$; Dunn's post hoc test, $p=7 \times 10^{-4}, 1.4 \times 10^{-3}$, $1)$. Overexpression of hTaoK2 ${ }^{\mathrm{WT}}$ and hTaoK2 ${ }^{\mathrm{A} 335 \mathrm{~V}}$, but not hTaoK2 ${ }^{\mathrm{A} 135 \mathrm{P}}$, fully rescued these behavioral defects. Together, these data reveal that Drosophila Tao and hTaoK2 are functionally conserved and regulate dendritic branching, the number of dynamic MTs and neuronal function in a kinase activitydependent manner, with a subset of ASD-linked hTaok2 mutations (A135P, P1022*) displaying impaired function in this system.

Tao function is required to maintain adult sensory neuron arborization, escape, and social behavior

ASDs are characterized by altered sensory perception and social behavior. To address whether developmental or acute loss of Tao function in sensory neurons is also affecting these ASD-related behaviors in flies, we first tested whether dendritic morphology of adult sensory neurons requires Tao function. Similarly to larval stages, developmental downregulation of Tao kinase resulted in an increase of dendritic growth and complexity in adult C4da neurons (Fig. 10A-D; unpaired $t$ test, Fig. 10B: $t_{(18)}=-6.515$, $p=3.991 \times 10^{-6}$; Fig. 10C: $t_{(18)}=-8.315, p=1.407 \times 10^{-7}$; Fig. 10D: unpaired $t$ test was conducted for each distance point, every $1 \mu \mathrm{m} ;{ }^{\star} p<0.05$ from 14 to $321 \mu \mathrm{m}$, total $\left.542 \mu \mathrm{m}\right)$. We then tested whether adult-specific loss of Tao function also resulted in dendritic defects by using temperature-sensitive Gal80 (Gal80 $\left.{ }^{\text {ts }}\right)$ to induce Tao ${ }^{\mathrm{RNAi}}$ expression $2 \mathrm{~d}$ after eclosion by shifting ani-

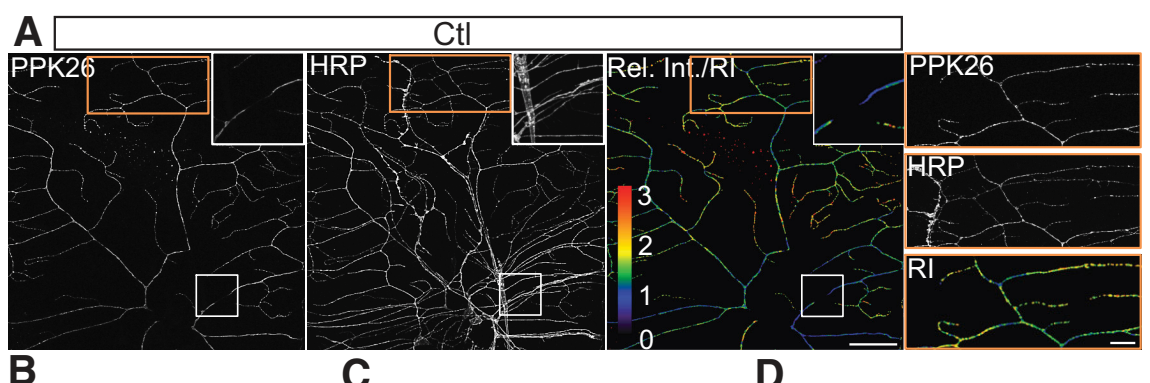

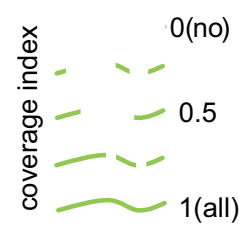

E

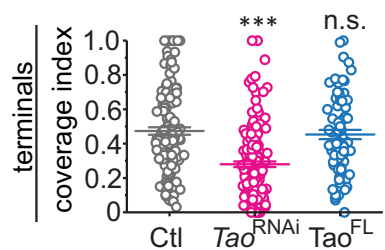

H CtI Tao RNAi TaoFL
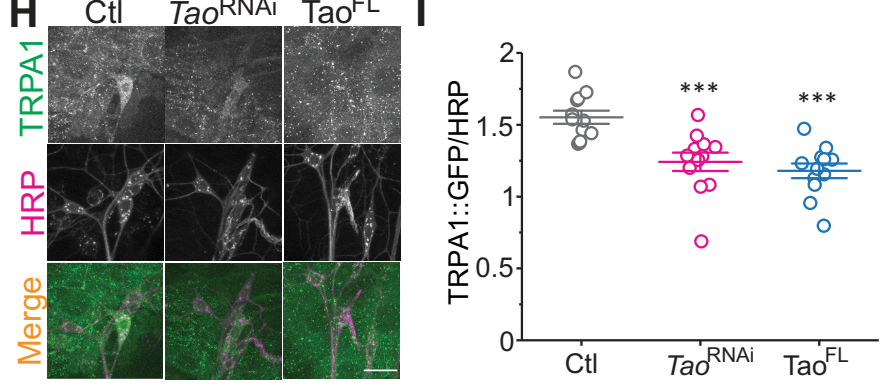

Figure 8. Manipulation of Tao activity affects levels and distribution of mechanosensory channels. A, Endogenous ppk26 immunoreactivity (magenta) was detected on dendrites of (4da neurons by immunohistochemistry under nonpermeabilizing conditions in WT (Ctl) C4da neurons. Anti-HRP signal labels the cell surface of all sensory neurons and was used for normalization. Relative intensity (RI) of ppk26 normalized to the HRP signal is shown. Enlarged white (main branches) and orange (terminals) boxes show that ppk26 immunoreactivity along the dendrites is not uniform. $\boldsymbol{B}$, The dendritic length from RI images was measured. The ppk26 coverage index was defined as the length of ppk26-positive dendrites divided by the corresponding total dendritic length. The ppk26 index in a single dendrite ranges from 0 (ppk26 RI signals could not be detected throughout the whole dendrite) to 1 (RI of ppk26 signals are detected throughout the whole dendrite). C, Quantifications comparing the ppk26 index of control (Ctl), Tao ${ }^{\mathrm{RNAi}}$, and $T a 0^{\mathrm{FL}}$ in C4da neuron main branches. $\boldsymbol{D}$, The overall RI of ppk26 in main branches was measured. $\boldsymbol{E}-\boldsymbol{G}$, Quantifications comparing the ppk26 index of $\mathrm{Ctl}, \mathrm{TaO}^{\mathrm{RNAi}}$, and $\mathrm{Ta}_{0}{ }^{\mathrm{FL}}$ in distal terminal branches. $\boldsymbol{E}$, The average ppk26 coverage index was shown. Each dot represents a terminal branch. $\boldsymbol{F}$, Distribution analysis of binned ppk26 coverage indexes for the indicated genotypes. G, The RI of ppk26 in terminal branches was measured and plotted for the indicated genotypes. Scale bar, 50 $\mu \mathrm{m} . \boldsymbol{C}, \boldsymbol{D}, n>40,40,35$ main branches from 8, 8, 7 neurons. $\boldsymbol{H}, \boldsymbol{J}, n>100,100,70$ terminals from 8, 8, 7 neurons. ${ }^{* *} p<0.01$, ${ }^{* * *} p<0.001$ (one-way ANOVA with Bonferroni correction). $\boldsymbol{H}$, Endogenously GFP-tagged TrpA1 channel expression $(+/$ $\operatorname{TrpA}^{\text {MI07645-GFSTF.O }}$ ) was detected by anti-GFP immunostaining in controls or with Tao ${ }^{\mathrm{RNAi}}$ and Tao ${ }^{\mathrm{FL}}$ expression in sensory neurons (21-7-Gal4 ). Anti-HRP immunoreactivity was used for normalization. TRPA1 is only expressed in C4da neurons. Scale bar, $20 \mu \mathrm{m}$. I, Statistical analysis of TrpA1 expression levels in somata of (4da neurons ( $n=12 /$ genotype). ${ }^{* * *} p<0.001$ comparing with Ctl (one-way ANOVA with Bonferroni correction).

mals from permissive $\left(18^{\circ} \mathrm{C}\right)$ to restrictive temperatures $\left(29^{\circ} \mathrm{C}\right)$. Interestingly, adult-specific loss of Tao function significantly increased dendritic complexity of C4da neurons as well, suggesting that Tao function in sensory neurons is required during development and in adults to maintain appropriate dendritic arborization (Fig. 10E-H; one-way ANOVA, Fig. 10F: $F_{(3,36)}=21.164$, $p=4.478 \times 10^{-8}$; Bonferroni post hoc test: Ctl 18 vs Ctl 29: 1 ; Ctl 18 vs Tao ${ }^{\mathrm{RNAi}} 18$ : 1 ; Ctl 29 vs Tao ${ }^{\mathrm{RNAi}} 29: 2.990 \times 10^{-6} ;$ Tao $^{\mathrm{RNAi}}$ 18 vs Tao $^{\mathrm{RNAi}} 29: 4.870 \times 10^{-7}$; Fig. $10 G: F_{(3,36)}=10.396, p=$ 
A

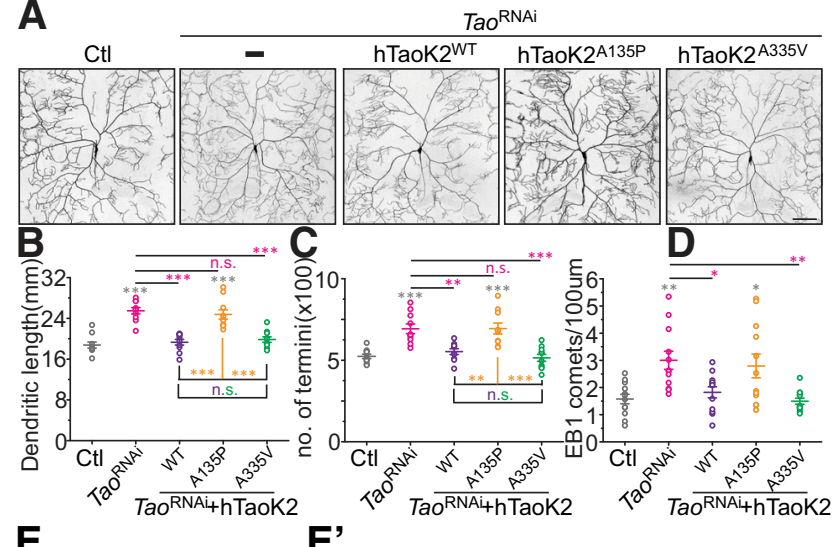

E

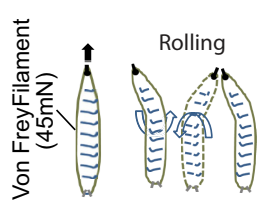

E'

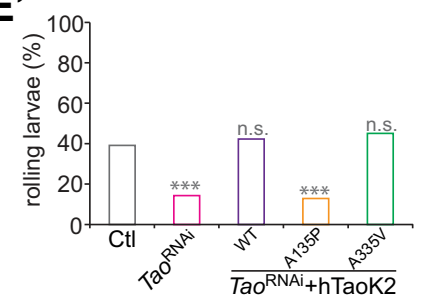

Figure 9. Human TaoK2, but not an ASD-linked variant, can functionally replace Drosophila Tao. $A$, Representative images showing dendritic morphology of C 4 da neurons after knockdown of Tao and rescue with hTaoK2 WT or A135P and A335V variants in C4da neurons. Scale bar, 100 $\mu \mathrm{m} . \boldsymbol{B}-\boldsymbol{D}$, Quantitative analysis of the total dendritic length $(\boldsymbol{B})$, number of terminals $(\boldsymbol{C})$, and number of EB1 comets/dynamic MTs (D) in $T a 0^{R N A i}$ and $T a 0^{R N A i} / \mathrm{hTa}$ ToK2-expressing (4da neurons $(n=10$ /genotypes for $\boldsymbol{A}-\boldsymbol{C} ; n=12 /$ genotypes for $\boldsymbol{D})$. ${ }^{*} p<0.05,{ }^{* *} p<0.01,{ }^{* * *} p<$ 0.001 (one-way ANOVA with Bonferroni post hoc test). $\boldsymbol{E}$, Schematic larval rolling response to mechanonociceptive stimulation using a $45 \mathrm{mN}$ von Frey filament. $\boldsymbol{E}^{\prime}$, Mechanonociceptive rolling responses of third instar larvae ( $96 \mathrm{~h} \mathrm{AEL)} \mathrm{after} \mathrm{(4da} \mathrm{neuron-specific} \mathrm{knockdown} \mathrm{of} \mathrm{Tao}$ and rescue with hTaoK2 WT, A135P, and A335V variants ( $n=73, n=70, n=72, n=70, n=$ 72). ${ }^{*} p<0.05,{ }^{* *} p<0.01,{ }^{* * *} p<0.001$ (Kruskal-Wallis test with Dunn's post hoc).

$4.543 \times 10^{-5}$; Bonferroni post hoc test: Ctl 18 vs Ctl 29: 1; Ctl 18 vs Tao ${ }^{\text {RNAi }} 18$ : 1 ; Ctl 29 vs Tao $^{\text {RNAi }} 29: 2.270 \times 10^{-4}$; Tao $^{\text {RNAi }} 18$ vs Tao ${ }^{\text {RNAi }}$ 29: 0.0036; Fig. 10H: two-way ANOVA, $F_{(3,5063)}=$ 308.6, $p<0.0001$; Tukey post hoc test: ${ }^{\star} p<0.05$, comparison between $\mathrm{Tao}^{\mathrm{RNAi}} 18$ vs Tao ${ }^{\mathrm{RNAi}} 29$ from 60 to $425 \mu \mathrm{m}$, every $5 \mu \mathrm{m})$.

We further tested negative geotaxis (escape behavior), locomotor activity, and social interaction in adult animals. We first analyzed heterozygous Tao mutant flies (Tao ${ }^{\text {eta/+ }}$ females), which displayed impaired negative geotaxis and altered locomotor activity in darkness (Fig. 11A-D; Fig. 11A: Mann-Whitney test, $U=97, p<0.0001$; Fig. 11C: Mann-Whitney test, $U=580, p=$ 0.366; Fig. $11 D$ : unpaired $t$ test, $\left.t_{(77)}=5.659, p<0.0001\right)$. We next assessed social interaction in heterozygous Tao mutant flies by counting the number of direct interactions during a $2 \mathrm{~min}$ period (approaches, lunges, tussles, wing threat, and initiation of courtship). Compared with controls, Tao ${ }^{\text {eta }}$ mutant heterozygous flies displayed strongly reduced social interactions (Fig. 11E; unpaired $t$ test, $\left.t_{(22)}=11.56, p<0.0001\right)$. As overall locomotion was not impaired (Fig. $11 B-D$ ), the observed social behavioral changes were not due to unspecific reduction of mobility.

Next, we asked whether (1) Tao-dependent behavioral changes were relying on sensory neuron function and (2) whether Tao was also required during adult stages for maintaining behavior. To test sensory or general neuronal Tao function, we expressed Tao ${ }^{\mathrm{RNAi}}$ using a somatosensory neuron-specific Gal4 line Gal4 ${ }^{5-40}$ (Cheng et al., 2010) or the pan-neuronal Gal4 ${ }^{\text {elav }}$, respectively. Both sensory neuron-specific as well as
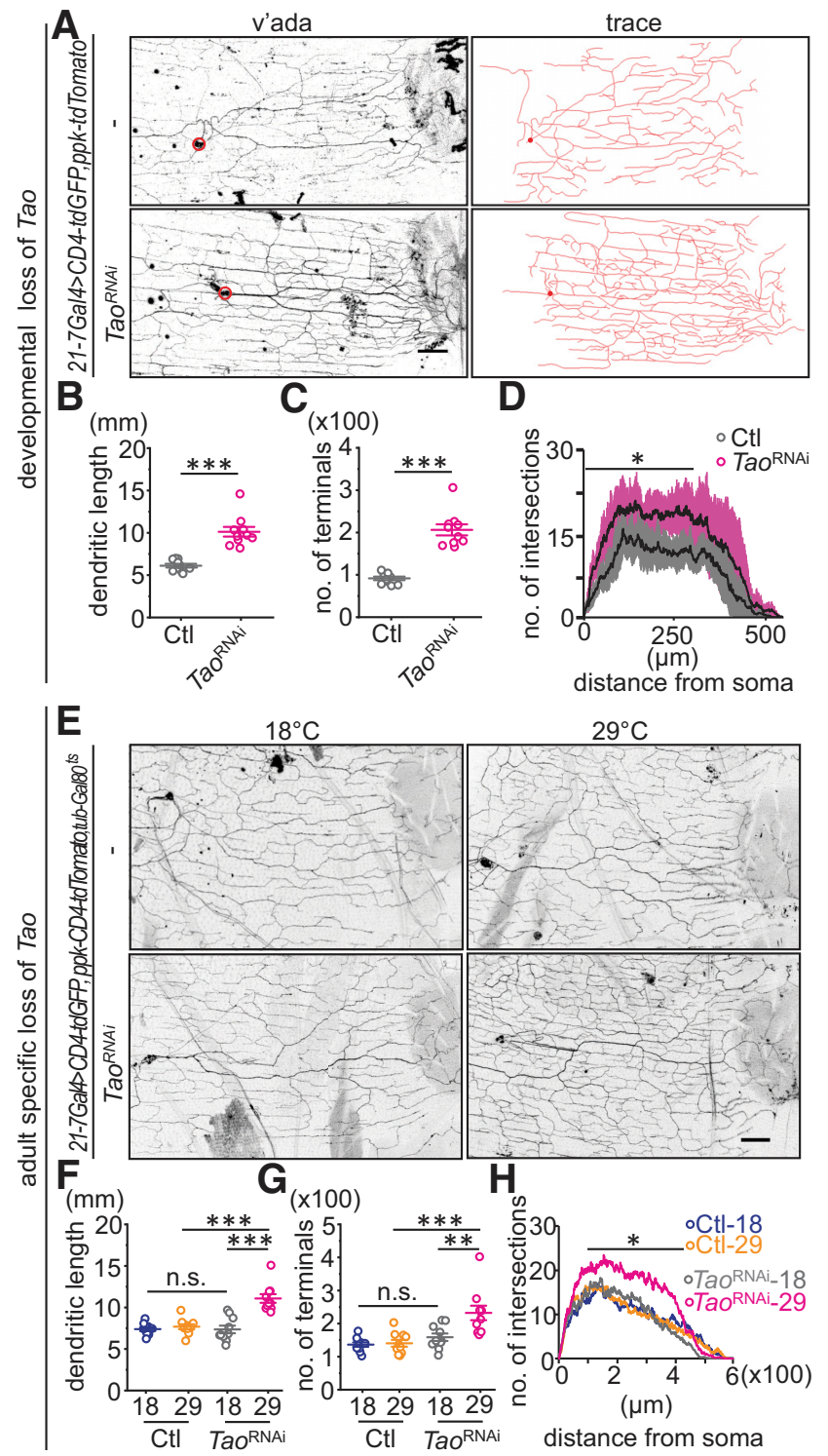

Figure 10. Developmental or adult-specific loss of Tao function affects dendritic arborization of adult sensory neurons. $\boldsymbol{A}-\boldsymbol{D}$, Developmental loss of Tao function results in dendritic overbranching in adult sensory (4da neurons. $A$, Dendritic morphology and traced dendrites of $v^{\prime}$ ada C4da neurons are shown. Red circles represent the soma. Scale bar, $50 \mu \mathrm{m}$. B-D, Quantification of dendritic length $(\boldsymbol{B})$, number of terminals $(\boldsymbol{C})$, and Sholl analysis $(\boldsymbol{D})(n=10$ / genotype). ${ }^{*} p<0.05,{ }^{* * *} p<0.001$ ( $t$ test). $\boldsymbol{E}-\boldsymbol{H}$, Adult-specific loss of Tao function results in dendritic overbranching. $\boldsymbol{E}$, Dendritic morphology of v'ada (4da neurons is shown with or without expression of $\mathrm{Ta}^{\mathrm{RNAi}}$ at the restrictive $\left(29^{\circ} \mathrm{C}\right)$ or permissive $\left(18^{\circ} \mathrm{C}\right)$ temperature, respectively. Scale bar, $50 \mu \mathrm{m}$. $\boldsymbol{F}-\boldsymbol{H}$, Quantification of dendritic length $(\boldsymbol{F})$, number of terminals $(\boldsymbol{G})$, and Sholl analysis $(\boldsymbol{H})\left(n=10 /\right.$ genotype). ${ }^{*} p<0.05,{ }^{* *} p<0.01,{ }^{* * *} p<0.001$ (one-way ANOVA with Bonferroni correction).

pan-neuronal loss of Tao recapitulated the defects observed in $\mathrm{TaO}^{\text {eta }}$ heterozygous mutants showing reduced negative geotaxis and social behavior (Fig. $11 F-H$; Kruskal-Wallis test with Dunn's post hoc test, Fig. $11 F: \mathrm{H}=59.28, p<0.0001,{ }^{*} \mathrm{UAS}-\mathrm{Tao}^{\mathrm{RNAi}} /+$, $540-G a l 4 /+$ and UAS-Tao ${ }^{\text {RNAi}} / 540-G a l 4 /$ UAS-hTaoK2 ${ }^{\text {WT }}$ vs UAS-Tao ${ }^{\mathrm{RNAi}} / 540-\mathrm{Gal} 4: p=0.0067, p=0.0015, p=0.0057$, ${ }^{\star * *} p=0.0005$; Fig. 11G: $\mathrm{H}=38.20, p<0.0001,{ }^{* *} p=0.0018$, ${ }^{* * * *} p<0.0001$; Fig. $11 \mathrm{H}: \mathrm{H}=30.60, p<0.0001,{ }^{* *} 540-\mathrm{Gal} 4 /+$ and UAS-Tao ${ }^{\text {RNAi }} / 540-$ Gal4/UAS-hTaoK2 ${ }^{\text {WT }}$ vs UAS-Tao ${ }^{\text {RNAi }}$ 540-Gal4: $\left.p=0.0015,0.0074,{ }^{* * *} p<0.0001\right)$. Coexpression of WT hTaok2 (hTaoK2 ${ }^{\mathrm{WT}}$ ) could fully rescue these behavioral defects, further showing that Tao function was strongly conserved 
and required in sensory neurons. We then tested adult-specific loss of Tao using temperature-sensitive Gal80 to induce Tao ${ }^{\text {RNAi }}$ expression $2 \mathrm{~d}$ after hatching by shifting them from permissive $\left(18^{\circ} \mathrm{C}\right)$ to restrictive temperatures $\left(29^{\circ} \mathrm{C}\right)$. Strikingly, Tao function was also required to maintain social behavior in adult flies (Fig. 11I; Kruskal-Wallis test with Dunn's post hoc test, $\mathrm{H}=64.57, p<0.0001$, UASTao $^{\text {RNAi }} /$ Elav-Gal4, TubGal80 ${ }^{\text {ts }} 18$ vs 29: $p=0.0062 ;$ UAS-Tao ${ }^{R N A i} /$ Elav-Gal4, TubGal80 ${ }^{\text {ts }} 29$ vs UAS-Tao ${ }^{R N A i} /$ Elav-Gal4, TubGal80 ${ }^{\text {ts }} / U A S-h$ TaoK2 ${ }^{W T} 29: p=0.0023$; UAS-Tao ${ }^{R N A i} /$ Elav-Gal4, TubGal80 ${ }^{\text {ts }} /$ UAShTaoK2 ${ }^{W T} 29$ vs UAS-Tao ${ }^{R N A i} /$ Elav-Gal4, TubGal80 ts $_{\text {UAS-hTaoK2 }}^{\text {Al35P }}$ 29: $p=$ 0.002). Moreover, WT hTaok2, but not the ASD-linked hTaok $2^{\text {A135P }}$, expression could rescue adult-specific social behavioral defects. Together, our behavioral and anatomical analysis shows that Tao function is required during development and in adulthood to maintain sensory neuron function and social behavior.

\section{Discussion}

\section{Tao function controls} dendritic arborization

We identified Tao kinase as a negative regulator of dendritic growth during Drosophila development. Tao controls dendritic arborization in a kinase activitydependent manner by limiting dynamic dendrite stability and growth, suggesting that kinase activity of Tao is precisely regulated to control appropriate dendrite arborization during development. In the absence of Tao, dynamic MT numbers and F-actin levels were strongly increased. Conversely, elevated Tao kinase activity reduced dendritic arborization, MT dynamics, and altered F-actin localization, implying a functional link between Taodependent cytoskeletal dynamics and dendritic arborization. We further found that this function of Tao is not limited to the developmental stage, but also required for maintenance of adult dendritic structures, suggesting that a continuous balance of Tao signaling is required.

Shaping of dendritic branching relies on intrinsic and extrinsic control of cellular pathways that ultimately regulate cytoskeletal dynamics (Jan and Jan, 2010; Dong et al., 2015). Vertebrate Tao kinases were previously reported to indirectly regulate MT and F-actin dynamics in vitro (Johne et al., 2008). In Drosophila S2 cells, Tao was shown to negatively regulate MT dynamics, and phosphorylated Tao was found to colocalize with plus-end tips of MTs (Liu et al., 2010), suggesting that active Drosophila Tao might interact with plus-end binding proteins to alter MT polymerization. We detected punctate structures of the active form of Tao in dendrites, where it might exert direct effects on MT dynamics. Recent studies have shown that MT dynamics are likely regulated locally in dendrites and contribute to dendrite growth (Nguyen et al., 2011; Ori-McKenney et al., 2012; Yalgin et
B

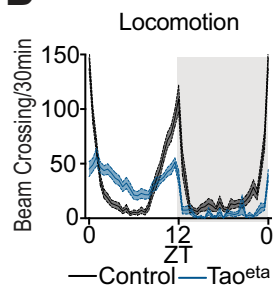

C

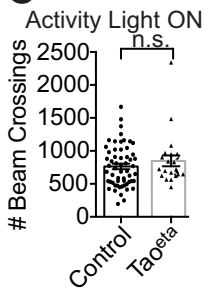

D

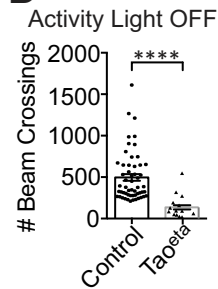

G

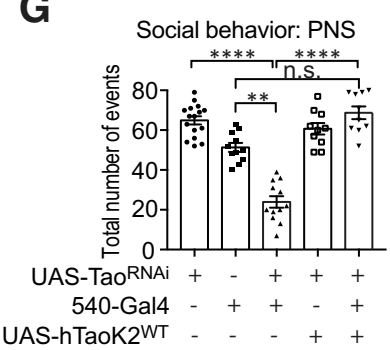

UAS-TaoRNAi +-+++ 540-Gal4 - + + - + UAS-hTaoK2WT $++$

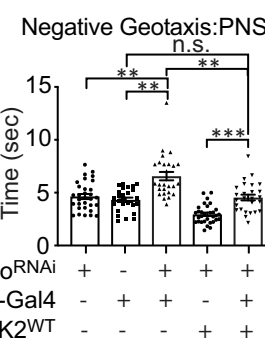

Social behavior: Panneuronal adults

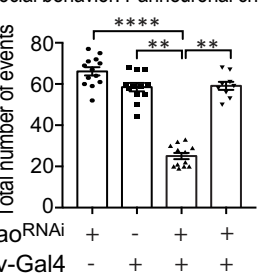
ic
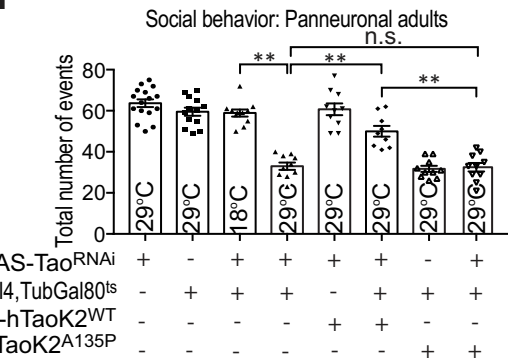

Figure 11. Tao activity in sensory neurons is required for adult motor reflexes and social interaction. $\boldsymbol{A}$, Performance of $T a 0^{\eta} /+$ (light gray bar) and control female flies (black bar) in negative geotaxis $(n=30, n=19)$. Data are mean \pm SE. ${ }^{* * *} p<0.001$ (Mann-Whitney test). $\boldsymbol{B}$, Locomotor activity of Control and Tao ${ }^{\text {eta/+ }}$ flies was monitored over $24 \mathrm{~h}$. Gray represents light-off we. $p<0.0001$ (Mann-Whitney test and test). E, Control and Tao female flies were for total number of social interactions in vials (average of number of total approaches, lunges, tussles, wing threat, and analyzed. Data are mean \pm SEM. ${ }^{* * * *} p<0.0001$ ( $t$ test). $\boldsymbol{F}$, Negative geotaxis and $(\boldsymbol{G})$ social interaction upon $T a 0^{R N A i}$ expression (PNS) using Gal ${ }^{-40} . F, n=31, n=26, n=27, n=31, n=26 . \mathbf{G}, n=16, n=12, n=$ (Kruskal-Wallis test with Dunn's post hoc). H Social behavior after pan-neuronal RNAi-mediated knockdown of Tao throughout development or $(\boldsymbol{I})$ in adults. Light gray bars represent genotypes with downregulated Tao. Black bars represent genetic control strains. Dark gray bars represent genetic rescue lines (UAS-TaoK2 ${ }^{\text {WT/A135P }}$, UAS-Ta0 ${ }^{R N A i}$ ). $\boldsymbol{H}, n=13, n=13, n=12, n=9 . \mathbf{G}, n=$ $16, n=13, n=11, n=10, n=9, n=10, n=10, n=10$ independent experiments (each with 8 flies)/genotype. Data are mean \pm SE. ${ }^{* *} p<0.01,{ }^{* * *} p<0.001$ (Kruskal-Wallis test with Dunn's post hoc).

al., 2015; Sanchez and Feldman, 2017). In this study, we found that Tao regulates MT dynamics in growing dendrites in a kinase activity-dependent manner affecting dynamic MT numbers but not polarity or speed. While the precise mechanism of how Tao controls dynamic MT numbers remains to be determined, MT interactors as well as transcriptional control have been implicated in linking MT dynamics and dendrite arborization: mutation of the MT severing enzyme Katanin p60-like 1 (Stewart et al., 2012) or the transcription factor FoxO (Sears and Broihier, 2016) results in a reduced number of dynamic MTs and, correspondingly, reduced dendritic branching. Similarly, the loss of minus-end stabilizing proteins, such as CAMSAP2, leads to reduced dendritic arborization and dynamic MT numbers (Yau et al., 2014). Additional studies in Drosophila and mammals have shown that Tao and its homolog Taok2 act through cytoskeletal regulators, including JNK (Moore et al., 2000; de Anda et al., 2012; Kapfhamer et al., 2012) and par-1/MARK (Timm et al., 2003; King et al., 2011) to control neuronal development. Tao kinase might be 
involved in phosphorylation of the MT-associated proteins Map $1 \mathrm{~b}$ and Tau via JNK and Par-1/MARK, respectively, which are important for MT stability (Kawauchi et al., 2005; Wang et al., 2007; Giacomini et al., 2018). However, we failed to detect a genetic link between Drosophila Tao and several of the abovementioned MT regulators, suggesting that a divergent downstream target might mediate Tao-regulated dendritic branching and MT dynamics in this system.

More recently, mammalian TaoK2 has been shown to be a substrate for the Hippo ortholog Mst3, and its activity is involved in regulating spine stability through various effectors, including myosin Va, septin 7, and RhoA (Ultanir et al., 2014; Yadav et al., 2017; Richter et al., 2019). This implies a functional role of TaoK2 in controlling F-actin dynamics as well, which is a critical factor for dendritic branching in C4da neurons (Ferreira et al., 2014; Stürner et al., 2019). In our study, we found that manipulation of Tao kinase activity altered actin localization and distribution, suggesting a contributing role of actin dynamics in Tao-regulated dendritic arborization.

\section{Tao kinase activity is required for sensory function}

Proper dendritic branch establishment during development is required for normal neuronal functions, and morphological abnormalities are strongly linked to neurodevelopmental disorders (Hall and Treinin, 2011; Kulkarni and Firestein, 2012; Koleske, 2013). However, perturbation of dendritic morphology can also result in different functional consequences depending on how the physiological properties of the neurons are affected. Even severe reduction of sensory dendrite structures as shown, for example, by loss of SREBP, can result in heightened behavioral responses (Ziegler et al., 2017). In our case, dendritic overgrowth induced by the loss of Tao in C4da neurons did not increase, but instead impaired mechanonociceptive responses. Consistently, we show that loss of Tao affected the localization and abundance of the mechanosensory channels ppk26 and TrpA1 in C4da neurons, both of which are required for mechanonociceptive responses (Zhong et al., 2012; Gorczyca et al., 2014; Guo et al., 2014; Mauthner et al., 2014). Our results thus hint at sensory defects linked to mechanosensory channel function. As Tao seems to regulate dendritic growth and cytoskeletal dynamics in all analyzed sensory neurons during development and in adults, it is likely that it affects the localization and/or function of other mechanosensory channels as well. This might include the gentle touch-sensitive Trp channel nompC, which is expressed in larval and adult mechanosensors and required for touch responses and locomotion behavior (Walker et al., 2000; Cheng et al., 2010; Yan et al., 2013). We could show that Tao function is required in somatosensory neurons of adult flies for normal escape behavior and social interaction. TRP channel function, including that of nompC and TrpV1, is strongly dependent on MTs, as proper gating requires direct $\mathrm{MT}$ interaction via their ankyrin repeats (Cheng et al., 2010; Prager-Khoutorsky et al., 2014; Zhang et al., 2015). Thus, it is feasible that Tao-dependent changes in dendritic MTs dynamics detrimentally affect TRP channel localization and function.

\section{Tao kinase function in sensory neurons links to ASD- relevant behaviors}

Recent studies have highlighted that sensory dysfunction, social interaction deficits, and anxiety-like behavior can arise by specific perturbation of ASD-linked genes in somatosensory neurons (Han et al., 2016; Orefice et al., 2016; Dawes et al., 2018). This suggests that sensory neuron dysfunction likely plays a direct role in at least some ASD-linked behaviors. Based on our analyses, sensory defects are likely also an important feature of TaoK2linked ASDs. Human TaoK2 could fully rescue anatomical and behavioral defects induced by the loss of Drosophila Tao at larval and adult stages, suggesting a high degree of functional conservation. Human TaoK2 has been strongly associated with ASDs due to its localization in the chromosomal $16 \mathrm{p} 11.2$ region, a major susceptibility locus for ASDs with a prevalence of $1 \%$ of patients carrying a corresponding microdeletion (Weiss et al., 2008). Recently, rare inherited and de novo mutations of hTaoK2 have been identified in ASD patients, suggesting a direct role for TaoK2 in ASD etiology (Richter et al., 2019). While the identified mutations in TaoK2 are heterogeneous in their effect on dendrite morphogenesis, it seems that deregulation of its localization and/or kinase activity have detrimental effects on dendrite morphogenesis and function. Reduction or $\mathrm{KO}$ of TaoK2 in mice resulted in reduced complexity of basal dendrites of neocortical neurons (de Anda et al., 2012; Richter et al., 2019), whereas ASD patient-derived mutations either resulted in exuberant (hTaok2 ${ }^{\mathrm{P} 1022 *}$ ) or reduced (hTaok $2^{\mathrm{A} 135 \mathrm{P}}$ ) dendritic arborization. Moreover, TaoK2 KO mice display social behavioral defects similar to other ASD mouse models.

Although the effects of Tao on dendritic arborization of Drosophila sensory neurons are apparently opposite to those observed in pyramidal neurons of the mouse cortex (de Anda et al., 2012), hTaoK2, but not its ASD-linked A135P mutant form, could fully rescue loss of Tao function-induced dendritic overbranching, dynamic MT numbers, and behavioral defects. It is possible that the signaling readout of Tao kinase function is inverted in invertebrates, as previously suggested by opposite effects of Tao activity on par-1/MARK function in Drosophila and mouse (Timm et al., 2003; King and Heberlein, 2011; King et al., 2011). However, ASDs and other neurodevelopmental disorders are displaying abnormal dendritic overgrowth or undergrowth (Lin and Koleske, 2010; Kulkarni and Firestein, 2012), both resulting in deleterious functional outcomes. For example, heterozygous $\mathrm{KO}$ mice of the ASD-linked tumor suppressor PTEN display exuberant dendritic arborization of cortical neurons and social behavioral defects in vivo (Huang et al., 2016). This suggests that appropriate growth regulation is essential for proper sensory and circuit function. Together, in accordance with previous reports, our study suggests that altered neuronal arborization and cytoskeletal dynamics result in sensory defects, which might be a common theme underlying ASDs and other neurodevelopmental disorders.

\section{References}

Chailangkarn T, Trujillo CA, Freitas BC, Hrvoj-Mihic B, Herai RH, Yu DX, Brown TT, Marchetto MC, Bardy C, McHenry L, Stefanacci L, Järvinen A, Searcy YM, DeWitt M, Wong W, Lai P, Ard MC, Hanson KL, Romero S, Jacobs B, et al. (2016) A human neurodevelopmental model for Williams syndrome. Nature 536:338-343.

Cheng LE, Song W, Looger LL, Jan LY, Jan YN (2010) The role of the TRP channel NompC in Drosophila larval and adult locomotion. Neuron 67: 373-380.

Dan I, Watanabe NM, Kusumi A (2001) The Ste20 group kinases as regulators of MAP kinase cascades. Trends Cell Biol 11:220-230.

Dankert H, Wang L, Hoopfer ED, Anderson DJ, Perona P (2009) Automated monitoring and analysis of social behavior in Drosophila. Nat Methods 6:297-303.

Dawes JM, Weir GA, Middleton SJ, Patel R, Chisholm KI, Pettingill P, Peck LJ, Sheridan J, Shakir A, Jacobson L, Gutierrez-Mecinas M, Galino J, Walcher J, Kühnemund J, Kuehn H, Sanna MD, Lang B, Clark AJ, Themistocleous AC, Iwagaki N, et al. (2018) Immune or genetic-mediated disruption of CASPR2 causes pain hypersensitivity due to enhanced primary afferent excitability. Neuron 97:806-822.e10. 
de Anda FC, Rosario AL, Durak O, Tran T, Gräff J, Meletis K, Rei D, Soda T, Madabhushi R, Ginty DD, Kolodkin AL, Tsai LH (2012) Autism spectrum disorder susceptibility gene TAOK2 affects basal dendrite formation in the neocortex. Nat Neurosci 15:1022-1031.

Domínguez-Iturza N, Bagni C (2019) A light touch on sociability. Cell 178:769-771.

Dong X, Shen K, Bülow HE (2015) Intrinsic and extrinsic mechanisms of dendritic morphogenesis. Annu Rev Physiol 77:271-300.

Ferreira T, Ou Y, Li S, Giniger E, van Meyel DJ (2014) Dendrite architecture organized by transcriptional control of the F-actin nucleator spire. Development 141:650-660.

Giacomini C, Koo CY, Yankova N, Tavares IA, Wray S, Noble W, Hanger DP, Morris JD (2018) A new TAO kinase inhibitor reduces tau phosphorylation at sites associated with neurodegeneration in human tauopathies. Acta Neuropathol Commun 6:37.

Gomez JM, Wang Y, Riechmann V (2012) Tao controls epithelial morphogenesis by promoting fasciclin 2 endocytosis. J Cell Biol 199:1131-1143.

Gorczyca DA, Younger S, Meltzer S, Kim SE, Cheng L, Song W, Lee HY, Jan LY, Jan YN (2014) Identification of Ppk26, a DEG/ENaC channel functioning with Ppk1 in a mutually dependent manner to guide locomotion behavior in Drosophila. Cell Rep 9:1446-1458.

Grueber WB, Jan LY, Jan YN (2002) Tiling of the Drosophila epidermis by multidendritic sensory neurons. Development 129:2867-2878.

Grueber WB, Jan LY, Jan YN (2003) Different levels of the homeodomain protein cut regulate distinct dendrite branching patterns of Drosophila multidendritic neurons. Cell 112:805-818.

Guo Y, Wang Y, Wang Q, Wang Z (2014) The role of PPK26 in Drosophila larval mechanical nociception. Cell Rep 9:1183-1190.

Hall DH, Treinin M (2011) How does morphology relate to function in sensory arbors? Trends Neurosci 34:443-451.

Han C, Jan LY, Jan YN (2011) Enhancer-driven membrane markers for analysis of nonautonomous mechanisms reveal neuron-glia interactions in Drosophila. Proc Natl Acad Sci U S A 108:9673-9678.

Han Q, Kim YH, Wang X, Liu D, Zhang ZJ, Bey AL, Lay M, Chang W, Berta T, Zhang Y, Jiang YH, Ji RR (2016) SHANK3 deficiency impairs heat hyperalgesia and TRPV1 signaling in primary sensory neurons. Neuron 92:1279-1293.

Hattori Y, Usui T, Satoh D, Moriyama S, Shimono K, Itoh T, Shirahige K, Uemura T (2013) Sensory-neuron subtype-specific transcriptional programs controlling dendrite morphogenesis: genome-wide analysis of abrupt and Knot/Collier. Dev Cell 27:530-544.

Hoyer N, Petersen M, Tenedini F, Soba P (2018) Assaying mechanonociceptive behavior in Drosophila larvae. Bio Protoc 8:e2736.

Hu C, Petersen M, Hoyer N, Spitzweck B, Tenedini F, Wang D, Gruschka A, Burchardt LS, Szpotowicz E, Schweizer M, Guntur AR, Yang CH, Soba P (2017) Sensory integration and neuromodulatory feedback facilitate Drosophila mechanonociceptive behavior. Nat Neurosci 20:1085-1095.

Huang WC, Chen Y, Page DT (2016) Hyperconnectivity of prefrontal cortex to amygdala projections in a mouse model of macrocephaly/autism syndrome. Nat Commun 7:13421.

Hummel T, Krukkert K, Roos J, Davis G, Klämbt C (2000) Drosophila Futsch/22C10 is a MAP1B-like protein required for dendritic and axonal development. Neuron 26:357-370.

Jan YN, Jan LY (2010) Branching out: mechanisms of dendritic arborization. Nat Rev Neurosci 11:316-328.

Jinushi-Nakao S, Arvind R, Amikura R, Kinameri E, Liu AW, Moore AW (2007) Knot/Collier and cut control different aspects of dendrite cytoskeleton and synergize to define final arbor shape. Neuron 56:963-978.

Johne C, Matenia D, Li XY, Timm T, Balusamy K, Mandelkow EM (2008) Spred1 and TESK1: two new interaction partners of the kinase MARKK/ TAO1 that link the microtubule and actin cytoskeleton. Mol Biol Cell 19:1391-1403.

Kapfhamer D, King I, Zou ME, Lim JP, Heberlein U, Wolf FW (2012) JNK pathway activation is controlled by Tao/TAOK3 to modulate ethanol sensitivity. PLoS One 7:e50594.

Kardash E, Bandemer J, Raz E (2011) Imaging protein activity in live embryos using fluorescence resonance energy transfer biosensors. Nat Protoc 6:1835-1846.

Kawauchi T, Chihama K, Nishimura YV, Nabeshima Y, Hoshino M (2005) MAP1B phosphorylation is differentially regulated by Cdk5/p35, Cdk5/ p25, and JNK. Biochem Biophys Res Commun 331:50-55.
Kim SE, Coste B, Chadha A, Cook B, Patapoutian A (2012) The role of Drosophila piezo in mechanical nociception. Nature 483:209-212.

King I, Heberlein U (2011) Tao kinases as coordinators of actin and microtubule dynamics in developing neurons. Commun Integr Biol 4:554-556.

King I, Tsai LT, Pflanz R, Voigt A, Lee S, Jäckle H, Lu B, Heberlein U (2011) Drosophila tao controls mushroom body development and ethanolstimulated behavior through par-1. J Neurosci 31:1139-1148.

Koleske AJ (2013) Molecular mechanisms of dendrite stability. Nat Rev Neurosci 14:536-550.

Konietzny A, Bär J, Mikhaylova M (2017) Dendritic actin cytoskeleton: structure, functions, and regulations. Front Cell Neurosci 11:147.

Kosmidis S, Botella JA, Mandilaras K, Schneuwly S, Skoulakis EM, Rouault TA, Missirlis F (2011) Ferritin overexpression in Drosophila glia leads to iron deposition in the optic lobes and late-onset behavioral defects. Neurobiol Dis 43:213-219.

Kulkarni VA, Firestein BL (2012) The dendritic tree and brain disorders. Mol Cell Neurosci 50:10-20.

Lee A, Li W, Xu K, Bogert BA, Su K, Gao FB (2003) Control of dendritic development by the Drosophila fragile X-related gene involves the small GTPase Rac1. Development 130:5543-5552.

Lee T, Luo L (2001) Mosaic analysis with a repressible cell marker (MARCM) for Drosophila neural development. Trends Neurosci 24:251254.

Lin WY, Williams C, Yan C, Koledachkina T, Luedke K, Dalton J, Bloomsburg S, Morrison N, Duncan KE, Kim CC, Parrish JZ (2015) The SLC36 transporter pathetic is required for extreme dendrite growth in Drosophila sensory neurons. Genes Dev 29:1120-1135.

Lin YC, Koleske AJ (2010) Mechanisms of synapse and dendrite maintenance and their disruption in psychiatric and neurodegenerative disorders. Annu Rev Neurosci 33:349-378.

Liu T, Rohn JL, Picone R, Kunda P, Baum B (2010) Tao-1 is a negative regulator of microtubule plus-end growth. J Cell Sci 123:2708-2716.

Mauthner SE, Hwang RY, Lewis AH, Xiao Q, Tsubouchi A, Wang Y, Honjo K, Skene JH, Grandl J, Tracey WD Jr (2014) Balboa binds to pickpocket in vivo and is required for mechanical nociception in Drosophila larvae. Curr Biol 24:2920-2925.

McCarthy SE, Makarov V, Kirov G, Addington AM, McClellan J, Yoon S, Perkins DO, Dickel DE, Kusenda M, Krastoshevsky O, Krause V, Kumar RA, Grozeva D, Malhotra D, Walsh T, Zackai EH, Kaplan P, Ganesh J, Krantz ID, Spinner NB, et al. (2009) Microduplications of 16p11.2 are associated with schizophrenia. Nat Genet 41:1223-1227.

McGuire SE, Le PT, Osborn AJ, Matsumoto K, Davis RL (2003) Spatiotemporal rescue of memory dysfunction in Drosophila. Science 302:17651768.

Moore TM, Garg R, Johnson C, Coptcoat MJ, Ridley AJ, Morris JD (2000) PSK, a novel STE20-like kinase derived from prostatic carcinoma that activates the c-jun $\mathrm{N}$-terminal kinase mitogen-activated protein kinase pathway and regulates actin cytoskeletal organization. J Biol Chem 275: 4311-4322.

Nguyen MM, Stone MC, Rolls MM (2011) Microtubules are organized independently of the centrosome in Drosophila neurons. Neural Dev 6:38.

Nguyen MM, McCracken CJ, Milner ES, Goetschius DJ, Weiner AT, Long MK, Michael NL, Munro S, Rolls MM (2014) $\gamma$-Tubulin controls neuronal microtubule polarity independently of Golgi outposts. Mol Biol Cell 25:2039-2050.

Orefice LL, Zimmerman AL, Chirila AM, Sleboda SJ, Head JP, Ginty DD (2016) Peripheral mechanosensory neuron dysfunction underlies tactile and behavioral deficits in mouse models of ASDs. Cell 166:299-313.

Orefice LL, Mosko JR, Morency DT, Wells MF, Tasnim A, Mozeika SM, Ye M, Chirila AM, Emanuel AJ, Rankin G, Fame RM, Lehtinen MK, Feng G, Ginty DD (2019) Targeting peripheral somatosensory neurons to improve tactile-related phenotypes in ASD models. Cell 178:867-886.e24.

Ori-McKenney KM, Jan LY, Jan YN (2012) Golgi outposts shape dendrite morphology by functioning as sites of acentrosomal microtubule nucleation in neurons. Neuron 76:921-930.

Ori-McKenney KM, McKenney RJ, Huang HH, Li T, Meltzer S, Jan LY, Vale $\mathrm{RD}$, Wiita AP, Jan YN (2016) Phosphorylation of $\beta$-tubulin by the Down syndrome kinase, Minibrain/DYRK1a, regulates microtubule dynamics and dendrite morphogenesis. Neuron 90:551-563.

Parrish JZ, Xu P, Kim CC, Jan LY, Jan YN (2009) The microRNA bantam functions in epithelial cells to regulate scaling growth of dendrite arbors in Drosophila sensory neurons. Neuron 63:788-802. 
Pflanz R, Voigt A, Yakulov T, Jäckle H (2015) Drosophila gene tao-1 encodes proteins with and without a Ste20 kinase domain that affect cytoskeletal architecture and cell migration differently. Open Biol 5:140-161.

Poon CL, Liu W, Song Y, Gomez M, Kulaberoglu Y, Zhang X, Xu W, Veraksa A, Hergovich A, Ghabrial A, Harvey KF (2018) A Hippo-like signaling pathway controls tracheal morphogenesis in Drosophila melanogaster. Dev Cell 47:564-575.e5.

Prager-Khoutorsky M, Khoutorsky A, Bourque CW (2014) Unique interweaved microtubule scaffold mediates osmosensory transduction via physical interaction with TRPV1. Neuron 83:866-878.

Richter M, Murtaza N, Scharrenberg R, White SH, Johanns O, Walker S, Yuen RK, Schwanke B, Bedürftig B, Henis M, Scharf S, Kraus V, Dörk R, Hellmann J, Lindenmaier Z, Ellegood J, Hartung H, Kwan V, Sedlacik J, Fiehler J,et al. (2019) Altered TAOK2 activity causes autism-related neurodevelopmental and cognitive abnormalities through RhoA signaling. Mol Psychiatry 24:1329-1350.

Sanchez AD, Feldman JL (2017) Microtubule-organizing centers: from the centrosome to non-centrosomal sites. Curr Opin Cell Biol 44:93-101.

Sato K, Hayashi Y, Ninomiya Y, Shigenobu S, Arita K, Mukai M, Kobayashi S (2007) Maternal Nanos represses hid/skl-dependent apoptosis to maintain the germ line in Drosophila embryos 104:7455-7460.

Satoh D, Sato D, Tsuyama T, Saito M, Ohkura H, Rolls MM, Ishikawa F, Uemura T (2008) Spatial control of branching within dendritic arbors by dynein-dependent transport of Rab5-endosomes. Nat Cell Biol 10:1164-1171.

Sears JC, Broihier HT (2016) FoxO regulates microtubule dynamics and polarity to promote dendrite branching in Drosophila sensory neurons. Dev Biol 418:40-54.

Soba P, Han C, Zheng Y, Perea D, Miguel-Aliaga I, Jan LY, Jan YN (2015) The Ret receptor regulates sensory neuron dendrite growth and integrin mediated adhesion. Elife 4:e05491.

Song W, Onishi M, Jan LY, Jan YN (2007) Peripheral multidendritic sensory neurons are necessary for rhythmic locomotion behavior in Drosophila larvae. Proc Natl Acad Sci U S A 104:5199-5204.

Stewart A, Tsubouchi A, Rolls MM, Tracey WD, Sherwood NT (2012) Katanin $\mathrm{p} 60$-like1 promotes microtubule growth and terminal dendrite stability in the larval class IV sensory neurons of Drosophila. J Neurosci 32:11631-11642.

Stone MC, Rao K, Gheres KW, Kim S, Tao J, La Rochelle C, Folker CT, Sherwood NT, Rolls MM (2012) Normal spastin gene dosage is specifically required for axon regeneration. Cell Rep 2:1340-1350.

Stürner T, Tatarnikova A, Mueller J, Schaffran B, Cuntz H, Zhang Y, Nemethova M, Bogdan S, Small V, Tavosanis G (2019) Transient localization of the Arp2/3 complex initiates neuronal dendrite branching in vivo. Development 146:dev171397.

Sugimura K, Yamamoto M, Niwa R, Satoh D, Goto S, Taniguchi M, Hayashi S, Uemura T (2003) Distinct developmental modes and lesion-induced reactions of dendrites of two classes of Drosophila sensory neurons. J Neurosci 23:3752-3760.

Sugimura K, Satoh D, Estes P, Crews S, Uemura T (2004) Development of morphological diversity of dendrites in Drosophila by the BTB-zinc finger protein abrupt. Neuron 43:809-822.

Tao J, Feng C, Rolls MM (2016) The microtubule-severing protein fidgetin acts after dendrite injury to promote their degeneration. J Cell Sci 129:3274-3281.

Timm T, Li XY, Biernat J, Jiao J, Mandelkow E, Vandekerckhove J, Man- delkow EM (2003) MARKK, a Ste20-like kinase, activates the polarityinducing kinase MARK/PAR-1. EMBO J 22:5090-5101.

Tracey WD Jr, Wilson RI, Laurent G, Benzer S (2003) painless, a Drosophila gene essential for nociception. Cell 113:261-273

Ultanir SK, Yadav S, Hertz NT, Oses-Prieto JA, Claxton S, Burlingame AL, Shokat KM, Jan LY, Jan YN (2014) MST3 kinase phosphorylates TAO1/2 to enable Myosin Va function in promoting spine synapse development. Neuron 84:968-982.

Walker RG, Willingham AT, Zuker CS (2000) A Drosophila mechanosensory transduction channel. Science 287:2229-2234.

Wang JW, Imai Y, Lu B (2007) Activation of PAR-1 kinase and stimulation of tau phosphorylation by diverse signals require the tumor suppressor protein LKB1. J Neurosci 27:574-581.

Weiss LA, Shen Y, Korn JM, Arking DE, Miller DT, Fossdal R, Saemundsen E, Stefansson H, Ferreira MA, Green T, Platt OS, Ruderfer DM, Walsh CA, Altshuler D, Chakravarti A, Tanzi RE, Stefansson K, Santangelo SL, Gusella JF, Sklar P, et al. (2008) Association between microdeletion and microduplication at 16p11.2 and autism. N Engl J Med 358:667-675.

Yadav S, Oses-Prieto JA, Peters CJ, Zhou J, Pleasure SJ, Burlingame AL, Jan LY, Jan YN (2017) TAOK2 kinase mediates PSD95 stability and dendritic spine maturation through Septin7 phosphorylation. Neuron 93:379-393.

Yalgin C, Ebrahimi S, Delandre C, Yoong LF, Akimoto S, Tran H, Amikura R, Spokony R, Torben-Nielsen B, White KP, Moore AW (2015) Centrosomin represses dendrite branching by orienting microtubule nucleation. Nat Neurosci 18:1437-1445.

Yan Z, Zhang W, He Y, Gorczyca D, Xiang Y, Cheng LE, Meltzer S, Jan LY, Jan YN (2013) Drosophila NOMPC is a mechanotransduction channel subunit for gentle-touch sensation. Nature 493:221-225.

Yau KW, van Beuningen SF, Cunha-Ferreira I, Cloin BM, van Battum EY, Will L, Schätzle P, Tas RP, van Krugten J, Katrukha EA, Jiang K, Wulf PS, Mikhaylova M, Harterink M, Pasterkamp RJ, Akhmanova A, Kapitein LC, Hoogenraad CC (2014) Microtubule minus-end binding protein CAMSAP2 controls axon specification and dendrite development. Neuron 82:1058-1073.

Zhang W, Cheng LE, Kittelmann M, Li J, Petkovic M, Cheng T, Jin P, Guo Z, Göpfert MC, Jan LY, Jan YN (2015) Ankyrin repeats convey force to gate the NOMPC mechanotransduction channel. Cell 162:1391-1403.

Zhao B, Meka DP, Scharrenberg R, König T, Schwanke B, Kobler O, Windhorst S, Kreutz MR, Mikhaylova M, Calderon de Anda F (2017) Microtubules modulate F-actin dynamics during neuronal polarization. Sci Rep 7:9583.

Zheng Y, Wildonger J, Ye B, Zhang Y, Kita A, Younger SH, Zimmerman S, Jan LY, Jan YN (2008) Dynein is required for polarized dendritic transport and uniform microtubule orientation in axons. Nat Cell Biol 10: 1172-1180.

Zhong L, Hwang RY, Tracey WD (2010) Pickpocket is a DEG/ENaC protein required for mechanical nociception in Drosophila larvae. Curr Biol 20:429-434.

Zhong L, Bellemer A, Yan H, Ken H, Jessica R, Hwang RY, Pitt GS, Tracey WD (2012) Thermosensory and nonthermosensory isoforms of Drosophila melanogaster TRPA1 reveal heat-sensor domains of a thermoTRP channel. Cell Rep 1:43-55.

Ziegler AB, Thiele C, Tenedini F, Richard M, Leyendecker P, Hoermann A, Soba P, Tavosanis G (2017) Cell-autonomous control of neuronal dendrite expansion via the fatty acid synthesis regulator SREBP. Cell Rep 21:3346-3353. 